Dausien, Bettina; Walgenbach, Katharina

\title{
Sozialisation von Geschlecht - Skizzen zu einem wissenschaftlichen Diskurs und Plädoyer für die Revitalisierung einer gesellschaftsanalytischen Perspektive
}

Dausien, Bettina [Hrsg.]; Thon, Christine [Hrsg.]; Walgenbach, Katharina [Hrsg.]: Geschlecht - Sozialisation

- Transformationen. Opladen ; Berlin ; Toronto : Verlag Barbara Budrich 2015, S. 17-50. - (Jahrbuch

Frauen- und Geschlechterforschung in der Erziehungswissenschaft; 11)

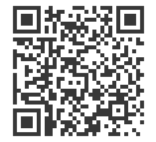

Quellenangabe/ Reference:

Dausien, Bettina; Walgenbach, Katharina: Sozialisation von Geschlecht - Skizzen zu einem wissenschaftlichen Diskurs und Plädoyer für die Revitalisierung einer gesellschaftsanalytischen Perspektive - In: Dausien, Bettina [Hrsg.]; Thon, Christine [Hrsg.]; Walgenbach, Katharina [Hrsg.]: Geschlecht - Sozialisation - Transformationen. Opladen; Berlin ; Toronto : Verlag Barbara Budrich 2015 , S. 17-50 - URN: urn:nbn:de:0111-pedocs-130297 - DOI: 10.25656/01:13029

https://nbn-resolving.org/urn:nbn:de:0111-pedocs-130297

https://doi.org/10.25656/01:13029

in Kooperation mit / in cooperation with:

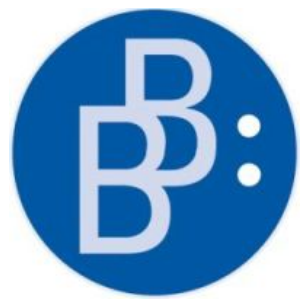

https://www.budrich.de

\section{Nutzungsbedingungen}

Gewährt wird ein nicht exklusives, nicht übertragbares, persönliches und beschränktes Recht auf Nutzung dieses Dokuments. Dieses Dokument ist ausschließlich für den persönlichen, nicht-kommerziellen Gebrauch bestimmt. Die Nutzung stellt keine Übertragung des Eigentumsrechts an diesem Dokument dar und gilt vorbehaltlich der folgenden Einschränkungen: Auf sämtlichen Kopien dieses Dokuments müssen alle Urheberrechtshinweise und sonstigen Hinweise auf gesetzlichen Schutz beibehalten werden. Sie dürfen dieses Dokument nicht in irgendeiner Weise abändern, noch dürfen Sie dieses Dokument für öffentliche oder kommerzielle Zwecke vervielfältigen, öffentlich ausstellen, aufführen, vertreiben oder anderweitig nutzen.

Mit der Verwendung dieses Dokuments erkennen Sie die Nutzungsbedingungen an.

\section{Terms of use}

We grant a non-exclusive, non-transferable, individual and limited right to using this document.

This document is solely intended for your personal, non-commercial use. Use of this document does not include any transfer of property rights and it is conditional to the following limitations: All of the copies of this documents must retain all copyright information and other information regarding legal protection. You are not allowed to alter this document in any way, to copy it for public or commercial purposes, to exhibit the document in public, to perform, distribute or otherwise use the document in public.

By using this particular document, you accept the above-stated conditions of use.

\section{Kontakt / Contact:}

\section{peDOCS}

DIPF | Leibniz-Institut für Bildungsforschung und Bildungsinformation

Informationszentrum (IZ) Bildung

E-Mail: pedocs@dipf.de

Internet: www.pedocs.de

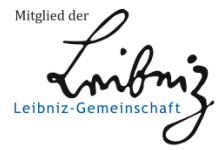


Bettina Dausien
Christine Thon
Katharina Walgenbach (Hrsg.)

11/2015

Jahrbuch Frauen- und Geschlechterforschung in der Erziehungswissenschaft

Geschlecht - Sozialisation - Transformationen 
Jahrbuch

Frauen- und Geschlechterforschung in der Erziehungswissenschaft

Redaktion

Jürgen Budde

Astrid Messerschmidt

Christine Thon

Katharina Walgenbach

Beirat

Birgit Althans

Sabine Andresen

Eva Breitenbach

Rita Casale

Bettina Dausien

Isabell Diehm

Hannelore Faulstich-Wieland

Edgar Forster

Edith Glaser

Carola Iller

Marita Kampshoff

Margret Kraul

Andrea Liesner

Susanne Maurer

Vera Moser

Inga Pinhard

Annedore Prengel

Barbara Rendtorff

Folge 11/2015 
Bettina Dausien

Christine Thon

Katharina Walgenbach (Hrsg.)

\section{Geschlecht - Sozialisation - Transformationen}

Verlag Barbara Budrich

Opladen • Berlin • Toronto 2015 
Bibliografische Information der Deutschen Nationalbibliothek

Die Deutsche Nationalbibliothek verzeichnet diese Publikation in der Deutschen Nationalbibliografie; detaillierte bibliografische Daten sind im Internet über

http://dnb.d-nb.de abrufbar.

Gedruckt auf säurefreiem und alterungsbeständigem Papier.

Alle Rechte vorbehalten.

C) 2015 Verlag Barbara Budrich, Opladen, Berlin \& Toronto

www.budrich-verlag.de

ISBN 978-3-8474-0668-6 (Paperback)

eISBN 978-3-8474-0854-3 (eBook)

Das Werk einschließlich aller seiner Teile ist urheberrechtlich geschützt. Jede Verwertung außerhalb der engen Grenzen des Urheberrechtsgesetzes ist ohne Zustimmung des Verlages unzulässig und strafbar. Das gilt insbesondere für Vervielfältigungen, Übersetzungen, Mikroverfilmungen und die Einspeicherung und Verarbeitung in elektronischen Systemen.

Umschlaggestaltung: disegno visuelle kommunikation, Wuppertal - www.disenjo.de Lektorat: Christine Berberich

Typographisches Lektorat: Ulrike Weingärtner, Gründau 


\section{Inhalt}

\section{Einleitung}

Bettina Dausien, Christine Thon, Katharina Walgenbach

Geschlecht - Sozialisation - Transformationen

\section{Essay}

Bettina Dausien, Katharina Walgenbach

Sozialisation von Geschlecht -

Skizzen zu einem wissenschaftlichen Diskurs und Plädoyer für

die Revitalisierung einer gesellschaftsanalytischen Perspektive

\section{Theoretische Analysen - Transformationen von Debatten}

\section{Jutta Hartmann}

Transformatorische Denkbewegungen -

Weiterentwicklung von Sozialisationstheorien im Anschluss an

Judith Butlers diskurstheoretisches Verständnis von Geschlecht

Barbara Rendtorff

Zugewinne und Fallen - aktuelle Veränderungen

in Geschlechtervorstellungen und ihre Probleme

Mechthild Bereswill, Gudrun Ehlert

Sozialisation im Kontext des Krisendiskurses über Jungen

Empirische Analysen -

Transformationen von Geschlechterverhältnissen

Sabine Klinger

Die (De-)Thematisierung von Geschlechterhierarchien im Verhältnis akademischer Sozialisationsprozesse und gesellschaftlicher Diskurse.

Eine qualitativ-rekonstruktive Analyse studentischer

Gruppendiskussionen

Monika Götsch

Paradoxien heteronormativer Sozialisation 
Inhalt

\section{Offener Teil}

Karin Flaake

Neue Konstellationen für Männlichkeitsentwürfe -

Potentiale einer in der Paarbeziehung geteilten Elternschaft

für Entwicklungsmöglichkeiten von Jungen und jungen Männern

Rhea Seehaus

(Un-)Ordnungen der familialen Sorge?

Mütter und die diskursive Figur des , aktiven Vaters ${ }^{6}$

Stefanie Leinfellner

Ambivalenzen und transformative Potenziale im Arbeitskontext

Wissenschaft: Zur Vereinbarkeit von Wissenschaft und Elternschaft in Dual-Career-Familien

\section{Tagungsbericht}

Anne-Dorothee Warmuth

„Erziehung - Gewalt - Sexualität“. Tagungsbericht zur Jahrestagung der Sektion Frauen- und Geschlechterforschung der DGfE an der Universität Paderborn (05./06. März 2015)

Verzeichnis der Autor_innen 209 
Essay 



\title{
Sozialisation von Geschlecht - Skizzen zu einem wissenschaftlichen Diskurs und Plädoyer für die Revitalisierung einer gesellschaftsanalytischen Perspektive
}

\author{
Bettina Dausien, Katharina Walgenbach
}

Ausgangspunkt des folgenden Essays ist die Beobachtung, dass sozialisationstheoretisches Denken in der erziehungswissenschaftlichen Geschlechterforschung seit längerem einen erheblichen Relevanzverlust erlitten hat. Dies ist umso erstaunlicher, als das Thema in den Anfängen der Frauen- und Geschlechterforschung wissenschaftlich, aber auch in der pädagogischen Praxis hohe Aufmerksamkeit hatte, ja, zu den konstitutiven Problemstellungen der Geschlechterforschung und der Frauenbewegung zählte. Heute scheint Geschlechtersozialisation dagegen ein Un-Thema geworden zu sein. Wir möchten in diesem Essay reflektieren, was es bedeutet, wenn der sozialisationstheoretische Blick in aktuellen Analysen der (erziehungswissenschaftlichen) Geschlechterforschung fehlt. Dafür verfolgen wir zwei miteinander verknüpfte Fragestellungen: Wie ist der Verlust sozialisationstheoretischer Fragen zu erklären? Und welche wichtigen Themen und Perspektiven geraten damit aus dem Blick?

Um der ersten Frage nachzugehen schauen wir, in Teil I des Essays, zunächst noch einmal zurück auf die Geschichte der erziehungswissenschaftlichen Geschlechterforschung. Ziel ist weniger eine genaue historische Rekonstruktion der Debatten um Geschlechtersozialisation als vielmehr die Entwicklung einer Argumentationsskizze, mit der wir den aktuellen Bedeutungsverlust des Themas plausibilisieren können. Wir entwickeln die These, dass unterschiedliche, auch konträre Strömungen der erziehungswissenschaftlichen Geschlechterforschung auf eine Weise ,zusammengespielt' haben, dass sich das Interesse auf die Analyse von Mikropraktiken und Diskursen sowie auf Prozesse der Individualisierung verlagert hat und dass damit sozialisationstheoretische Fragen gewissermaßen an den Rand gerückt sind. Dazu hat wesentlich auch die Rezeption des Individualisierungsansatzes in der Erziehungswissen- 
schaft beigetragen, bei der die gesellschaftsanalytische Dimension gewissermaßen ,weggekürzt' wurde und ,Individualisierung ' ohne das Konzept der Vergesellschaftung auszukommen scheint.

Der Rückblick in die Geschichte bereitet auch die Bearbeitung der zweiten Frage vor, denn er erinnert daran, dass das Sozialisationsparadigma durchaus unterschiedliche Forschungsrichtungen umfasst. Die Frage, was mit dem sozialisationstheoretischen Blick verlorengeht, kann nun, in Teil II des Essays, differenzierter beantwortet werden. Unser Anliegen ist es zu zeigen, dass insbesondere solche Ansätze aus dem Blick geraten sind, die Vergesellschaftung im Zusammenhang mit sozialen Ungleichheitsstrukturen und historisch-gesellschaftlichen Geschlechterverhältnissen auf der Strukturebene thematisieren.

Auf die problematische Ausblendung gesellschaftlicher Strukturzusammenhänge aus der Geschlechter(sozialisations)forschung haben einzelne Autorinnen wie Bettina Heintz und Eva Nadai (1998) oder Helga Bilden (2006) bereits vor einigen Jahren hingewiesen, ihre Argumente wurden allerdings nicht systematisch im Rahmen der Forschungen zu Sozialisation und Geschlecht und auch nicht in anderen erziehungswissenschaftlichen Diskursen aufgegriffen. Aktuelle Gesellschaftsdiagnosen zur Neuordnung von Ökonomie, Staat und Privatsphäre und deren Folgen für eine Transformation der Geschlechterverhältnisse bleiben damit tendenziell außerhalb erziehungswissenschaftlicher Reflexion ${ }^{1}$. Welche Bedeutung diese Ansätze für eine sozialisationstheoretische und erziehungswissenschaftliche Geschlechterforschung haben, diskutieren wir in Teil II. Umgekehrt erscheinen uns aber auch sozialisationstheoretische Perspektiven in aktuellen sozialwissenschaftlichen Gesellschaftsanalysen zu diesen Transformationsprozessen ein Forschungsdesiderat.

Wir denken, dass die in der jüngeren erziehungswissenschaftlichen Geschlechterforschung herrschende Distanz gegenüber kontemporären Analysen zur Transformation der Geschlechterverhältnisse auf der Makroebene durch eine Wiederaufnahme der sozialisationstheoretischen Perspektive aufgebrochen und korrigiert werden könnte. Vor diesem Hintergrund plädieren wir für eine Revitalisierung gesellschaftsanalytischer Perspektiven in der erziehungswissenschaftlichen Sozialisations- und Geschlechterforschung.

Die hier angesprochenen Themen sind weitgespannt und können im Format eines Essays gewiss nicht in angemessener Differenziertheit behandelt werden. Aktuelle wissenschaftliche Diskurse über Neoliberalismus, Postfordismus und neue Formen des Kapitalismus sind vielfältig, differenziert, oft auch überpointiert und unscharf, und gleiches gilt für Debatten über neue Formen der Subjektivierung und Identitätsformationen. Wir können diese Diskussionen hier nicht annähernd systematisch aufarbeiten - und schon gar keine schlüssige

1 Die Jahrbücher der Frauen- und Geschlechterforschung können hier als Ausnahme für die Erziehungswissenschaft angeführt werden (Borst/Casale 2007; Casale/Forster 2011; Moser/Pinhard 2010; Moser/Rendtorff 2012; siehe auch Walgenbach/Stach 2015). Sie fokussierten allerdings nicht dezidiert Fragen der Sozialisationsforschung und -theorie. 
Konzeption zu ihrer Verknüpfung vorlegen. Das ist jedoch auch nicht der Anspruch des Essays. Uns geht es darum, die Debatte um diese Fragen wieder neu anzustoßen. Es ist die ,alte' sozialisationstheoretische Fragestellung, auf die es womöglich keine befriedigenden Antworten gibt, die aber dennoch gestellt sein soll, ja muss, weil die gesellschaftlichen Verhältnisse sich in einem Ausmaß geändert haben, dass auch das analytische Instrumentarium der Geschlechter(sozialisations)forschung nicht fraglos beibehalten werden kann. Was an seine Stelle tritt oder treten sollte, ist eine offene Frage.

\section{Teil I}

\section{1 ,Geschlechtersozialisation' - Skizze zur Karriere eines wissenschaftlichen Paradigmas}

Das wissenschaftliche Konzept der Sozialisation ist in seiner Entstehungs- und Wirkungsgeschichte eng mit gesellschaftlichen Transformationsprozessen verknüpft: Dies gilt für sein erstes Auftauchen am Ende des 19. Jahrhunderts ebenso wie für spätere Konjunkturen, etwa im Kontext der Reformierung der Sozialwissenschaften nach dem Zweiten Weltkrieg (vgl. Veith 1996: 21ff., 36ff.). Es gilt auch für die Herausbildung des Konzepts der, geschlechtsspezifischen Sozialisation', das in den 1970er Jahren bedeutsam wurde. Die theoretische Grundfigur des Sozialisationskonzepts besteht darin, die Vergesellschaftung der Individuen und die Individualisierung des Sozialen als dialektischen Prozess zusammenzudenken, und zwar in der Dimension der Lebensspanne individueller Existenz oder, anders gesagt, in der Perspektive individueller Biographien.

Der von Émile Durkheim Ende des 19. Jahrhunderts erstmals formulierte Begriff Sozialisation enthielt gewissermaßen die soziologische Antwort auf die Frage, wie und unter welchen Bedingungen arbeitsteilige moderne Gesellschaften Integration und Kohärenz herstellen können, wenn traditionelle Moralvorstellungen und äußere Zwänge ihre Bindungskraft verlieren und die Lebensführung ihrer Mitglieder zunehmend kontingent und individualisiert wird. Durkheims Erklärung lenkte den Blick auf eine neue Form der Vergesellschaftung, auf die Internalisierung gesellschaftlicher Regeln und Strukturen, die zu einer Art Kollektivbewusstsein, einem Gemeinsinn oder einer „organischen Solidarität" führt. Diese bleibt jedoch bis zu einem gewissen Maße prekär und offen, da sie eben nicht mehr durch Religion oder äußere Gewalt „mechanisch“ 
erzwungen werden kann, sondern vom kollektiven Handeln der Individuen abhängig geworden ist (vgl. Durkheim [1893] 1977; Veith 1996: 114ff.).

Den Gedanken der Internalisierung oder Inkorporation ,äußerer' gesellschaftlicher Strukturen in die ,innere' Struktur der handelnden Subjekte haben später mit unterschiedlichen Akzentsetzungen Norbert Elias (1976) mit seinen Studien zum ,Prozess der Zivilisation“ und Pierre Bourdieu (1987) mit seinem Konzept des Habitus theoretisch und empirisch weitergeführt. Beide Ansätze zielen darauf ab, die Dichotomie von Individuum versus Gesellschaft, die historisch und theoriearchitektonisch gesehen für das Sozialisationsparadigma konstitutiv ist, zu überwinden (vgl. Bauer 2011: 107-120). Dieser Anspruch wird später auch in der Geschlechterforschung aufgenommen (s.u.).

Das ursprüngliche soziologische Interesse galt somit der theoretischen Erfassung des Zusammenhangs zwischen der Transformation der (europäischen) Gesellschaften zu modernen industrie-kapitalistisch wirtschaftenden, national organisierten Staaten einerseits und andererseits der Idee und empirischen Formation eines (männlichen) bürgerlichen Subjekts, das sich in diesen Strukturen herausbildet und zu einem handlungs- und leistungsfähigen, moralisch verpflichteten Mitglied heranwächst, zugleich aber auch seine je individuelle Persönlichkeit und Biographie ausbildet. ${ }^{2}$

\section{1 „, Geschlechtersozialisation“- Aufbruch und neue Perspektiven in den 1970er Jahren}

Dass dieses Subjekt ein Geschlecht hat oder, genauer, durch die in einer historisch-konkreten Gesellschaft herrschenden Geschlechterverhältnisse zu einem geschlechtlich positionierten und identifizierten Subjekt, zu ,Mann“ und ,Frau“ wird, war eine Erkenntnis, die erst Jahrzehnte später in der Frauenbewegung und der sich herausbildenden Frauen- und Geschlechterforschung thematisiert wurde. Simone de Beauvoirs vielzitierter Satz: „Man kommt nicht als Frau zur Welt, man wird es“ ([1949] 1951: 265), steht paradigmatisch für diese Einsicht.

Auch sie ist wiederum im Zusammenhang mit gesellschaftlichen Transformationsprozessen zu sehen, die zum Brüchigwerden der bis dahin dominanten Geschlechterordnung beitragen ${ }^{3}$ : mit dynamisierten Entwicklungen der Arbeitsmärkte ab den 1950er Jahren und dem steigenden Bedarf an qualifizierten

2 Zur Genese sozialisationstheoretischen Denkens vgl. die umfassende Arbeit von Veith (1996), zur Entwicklung der Debatte um Geschlechtersozialisation vgl. ausführlicher Dausien 1999, 2006; Bilden/Dausien 2006.

3 Die folgenden Stichworte lassen notgedrungen viele wichtige Differenzierungen aus, vor allem den Aspekt der Klassenlage, die nationalen Besonderheiten, Migrationsprozesse und die gesamte Entwicklung in den sozialistischen Nachkriegsgesellschaften Osteuropas. Sie sollen hier dennoch genannt werden, um das abstrakte Argument wenigstens mit einigen wenigen historischen Assoziationen zu verbinden und anschaulicher zu machen. 
Arbeitskräften; mit den Bildungsreformen, die seit den 1960er Jahren in den meisten westeuropäischen Gesellschaften politisch in Gang gesetzt und sehr rasch im Alltag wirksam werden; mit der Erosion und zunehmenden Kritik der im Nationalsozialismus geltenden Normen und Werte und dem Entstehen neuer politischer Ansätze zur Demokratisierung sowie neuer kultureller Orientierungen in den nachwachsenden Generationen; schließlich mit den konkreten biographischen Erfahrungen von Frauen (und Männern) im Nationalsozialismus und im Krieg, die jene vermeintlich traditionelle Geschlechterordnung bereits vielfach ad absurdum geführt hatten und einer schlichten Restauration in den Familien ebenso wie in der Gesellschaft entgegenstanden. Die sogenannte Studentenbewegung, die Frauenbewegung und andere neue soziale Bewegungen, die sich in den 1960er und 1970er Jahren bilden, sind politischer und kultureller Ausdruck einer gesellschaftlichen Transformation, die - neben der Klassenfrage - ganz wesentlich die Geschlechterfrage tangiert, ja durch sie vorangetrieben wird. Insbesondere zunehmend gut ausgebildete Frauen fordern jetzt nicht nur gleiche Rechte und Teilhabe, sondern machen sie immer öfter auch in der Alltagspraxis geltend. ${ }^{4}$ Sie kritisieren offen die bestehenden Machtverhältnisse zwischen den Geschlechtern sowohl auf der Ebene persönlicher Beziehungen als auch in ihrer institutionalisierten Struktur, die sich u.a. in den Schulen und Universitäten zeigt. In diesem vielschichtigen gesellschaftlichen Kontext, der als Modernisierungsprozess interpretiert werden kann, zugleich aber bereits Ansätze zu dessen Kritik enthält, ist auch die Diskussion um Geschlechtersozialisation zu verorten, die sich als interdisziplinäre Debatte in den 1970er Jahren etabliert.

\subsection{Etablierung im wissenschaftlichen Feld}

Im deutschsprachigen Raum gewinnt das Konzept der geschlechtsspezifischen (wie auch der klassenspezifischen) Sozialisation vornehmlich in soziologischen und erziehungswissenschaftlichen Diskursen rasch an Bedeutung; psychologische, kulturanthropologische und historische Forschungen werden zu seiner Stützung herangezogen. Das Konzept eröffnet die theoretisch begründete Möglichkeit, Geschlecht nicht mehr als ,Naturtatsache' zu behandeln, die pädagogisch allenfalls geformt und verfeinert werden kann (wie Geschlecht in der Pädagogik lange Zeit konzipiert war), sondern als ,soziale Tatsache“ (Durkheim). Es lenkt den Blick auf die gesellschaftliche Herstellung sozialer Rollen und vergeschlechtlichter Identitäten. „Wir werden nicht als Mädchen geboren - wir werden dazu gemacht", lautet der programmatische Titel einer

4 In einer rekonstruktiven Studie hat Christine Thon (2008) untersucht, wie solche Erfahrungen in den Lebensentwürfen und -praxen von Frauen über Generationen hinweg tradiert und transformiert werden. 
zeitgenössischen Publikation (Scheu 1977), die mit nicht unbedeutender Akzentverschiebung Simone de Beauvoirs Satz aufgreift (vgl. Dausien 1999: 225ff.).

Die sozialisationstheoretische Sichtweise bringt auch ein erhebliches Potenzial für empirische Forschung mit sich: Es werden neue Forschungsfragen und ganze Forschungsprogramme auf die Tagesordnung gesetzt (vgl. Hurrelmann/Ulich 1980) sowie neue Methoden ihrer Bearbeitung gesucht und entwickelt, Ansätze der quantitativen Sozialforschung ebenso wie die ab Mitte der 1970er Jahre rasch bedeutsam werdenden qualitativen Methoden und Konzepte der Methodenkombination. Das Sozialisationsparadigma ist in dieser Phase dabei nicht unwesentlich an der Transformation der geisteswissenschaftlichen Pädagogik in eine modernisierte, empirisch orientierte Erziehungswissenschaft beteiligt.

Das Sozialisationskonzept ist, insbesondere im Kontext der Frauenbewegung, auch mit dem Anspruch der Kritik an bisherigen wissenschaftlichen Sichtweisen und Forschungsansätzen verbunden. Geschlechtersozialisation gehört in den 1970er und 1980er Jahren zu jenen Themen, die sich zu Kristallisationspunkten im Aufbau einer kritischen Frauenforschung und feministischen Wissenschaft entwickeln. In den 1980er Jahren entsteht eine Vielzahl von Studien und theoretischen Diskussionen zum Thema Geschlechtersozialisation, die über die fachwissenschaftlichen Grenzen hinaus wahrgenommen werden und das Thema breit in den Erziehungs- und Sozialwissenschaften verankern. Diese Phase kann deshalb als Hochzeit des Konzepts der Geschlechtersozialisation bezeichnet werden.

Dies soll aber nicht darüber hinwegtäuschen, dass der Ansatz auch Probleme hat und keineswegs ein in sich schlüssiges, theoretisch konsistentes Paradigma darstellt. Im Gegenteil, die in den 1980er und 1990er Jahren an den Universitäten gut etablierte Sozialisationsforschung bezieht einen Großteil ihrer Integrationskraft aus dem Umstand, dass ,Sozialisation“ eine sehr abstrakte Überschrift für eine komplexe Problemstellung ist, die in konkreten Forschungskonzepten recht unterschiedlich ausbuchstabiert werden kann. Das gilt auch für die Forschungen zu Geschlechtersozialisation. Mit der gemeinsamen begrifflichen Klammer werden durchaus diskrepante, ja widersprüchliche Forschungsansätze zusammengefasst: Da finden sich psychoanalytisch ausgerichtete Ansätze, die in erster Linie nach der Dynamik der frühkindlichen Formation von Geschlechtsidentität fragen und diese als Reproduktion der geschlechtlichen Arbeitsteilung moderner Gesellschaften interpretieren (z.B. Chodorow 1985), neben Ansätzen, die sich im soziologischen Sinn um eine Analyse der widersprüchlichen Vergesellschaftungsprozesse bemühen und Geschlechterverhältnisse im Kontext gesellschaftlicher Strukturen und ihrer wechselseitigen Dynamiken verorten. Im deutschsprachigen Feld sind das vor allem die Arbeiten von Regina Becker-Schmidt und Gudrun-Axeli Knapp, die 
im Anschluss an die Kritische Theorie von einer konstitutiven Widerspruchsstruktur zwischen den gesellschaftlichen Sphären Produktion und Reproduktion ausgehen und daraus strukturell ambivalente Subjektformationen ableiten und empirisch rekonstruieren - und dabei die Tradition nicht-dualistischen, dialektischen Denkens in der Sozialisationsforschung prominent vertreten (vgl. stellvertretend Becker-Schmidt 1987; Knapp 1990).

Neben solchen, theoretisch elaborierten Ansätzen gibt es schließlich eine Vielzahl empirischer Forschungen, die einzelne Aspekte untersuchen und in einem sozialisationstheoretischen Rahmen verankern, z.B. Studien zur unterschiedlichen Behandlung, weiblicher' und ,männlicher' Kinder beim Stillen, im Kindergarten oder im Schulunterricht, zur Darstellung von ,Mädchen“ und ,Jungen' oder ,Frauen“ und ,Männern“ in Bilderbüchern, Schulbüchern, Talkshows oder in der Werbung (vgl. Schmerl 2006b).

Mit solchen Einzelstudien, die bis heute in der psychologischen oder erziehungswissenschaftlichen Geschlechterforschung durchaus üblich sind, werden einerseits bis dahin wenig beachtete Momente des komplexen Sozialisationsgeschehens identifiziert, andererseits führen sie kaum zu einem besseren theoretischen Verständnis des Zusammenhangs zwischen Sozialisation und Geschlecht. ${ }^{5}$ Sie tragen vielmehr, zugespitzt gesagt, mit dazu bei, den Dualismus ,weiblich/männlich` zu wiederholen und zu fixieren. Eben dieses Problem der Reifizierung der Geschlechterdifferenz durch die Frauenforschung selbst wird kurze Zeit später zentraler Ansatzpunkt der (Selbst-)Kritik.

\subsection{Geschlechtersozialisation als Perspektive in der politischen und pädagogischen Praxis}

Zunächst ist jedoch ein Blick über die Grenzen der wissenschaftlichen Debatte hinaus angezeigt. Der Aufschwung des Sozialisationsparadigmas in den 1970er und 1980er Jahren geht einher mit und wird auch getragen von einer breiteren gesellschaftlich-kulturellen Thematisierung der Geschlechterfrage, keineswegs nur in der Frauenbewegung im engeren Sinn. Die Idee, geschlechtsspezifischer' Sozialisation bietet Ansatzpunkte für eine kritisch-emanzipatorische Bildungs- und Erziehungspraxis, etwa für Konzepte einer parteilichen Mädchen- und Frauenbildung sowie für die Gestaltung der familialen

5 Helga Bildens programmatischer Entwurf, solche Befunde in einem aneignungstheoretischen Rahmen zu integrieren (Bilden 1980), wird nicht systematisch ausgearbeitet und von der Autorin selbst mehrfach kritisch reformuliert (Bilden 1991, 2006; vgl. auch Schmerl 2006a). 
Erziehung, die den kritisierten Geschlechterstereotypen ${ }^{6}$ durch ,gegengeschlechtliche ' Angebote ${ }^{7}$ oder, geschlechtsneutrale“ Interventionen gegensteuern sollten.

In dieser historischen Konstellation wird das Sozialisationskonzept zu einem Argument transformiert, das in pädagogischen Kontexten wie Schulen, Kindergärten, Universitäten oder der Bildungspolitik durchaus in seiner Sprengkraft erkannt und deshalb nicht nur positiv, sondern auch skeptisch aufgenommen wird: Das bestehende Geschlechterverhältnis oder, wie es im zeitgenössischen Jargon häufig heißt, die ,Geschlechterrollen' sind weder , angeboren" noch kulturell festgeschrieben, sondern Ergebnis historisch-gesellschaftlicher Prozesse. Sie werden ,gemacht' und - so die pädagogische Hoffnung - können deshalb auch verändert werden.

Diese Idee wird zwar in der Folge vordergründig enttäuscht, da sich die intendierten Effekte zumindest nicht unmittelbar einstellen, dennoch wird der Sozialisationsgedanke nachhaltig vom gesellschaftlichen Diskurs aufgenommen. In der pädagogischen Praxis und in der Bildungspolitik, in neuen Formen der ,Frauenförderung ' und später in der Gleichstellungspolitik ist das Konzept bzw. Argument der Geschlechtersozialisation nicht wegzudenken. Es wird zumeist ohne nähere Differenzierung und Explikation - als Erklärungsmuster für soziale Ungleichheit ebenso wie für pädagogische Probleme herangezogen und erfüllt ganz offensichtlich sehr gut die Funktion als gesellschaftliches Deutungsmuster. (Nebenbei gesagt, tritt das Argument ,Sozialisation' damit, funktional betrachtet, nicht selten an die Stelle seines vermeintlichen Gegenspielers: Es löst das Argument ab, Geschlecht sei eine biologische Disposition.)

\subsection{Kritik und Bedeutungsverlust: der Sozialisationsansatz seit den 1990er Jahren}

Im Unterschied dazu beginnt im wissenschaftlichen Kontext, noch während die Konjunktur der Sozialisationsforschung anhält, eine kritische Debatte, die Anfang der 1990er Jahre zu einer breiteren Diskussion und schließlich zur Ab-

6 Das Konzept der Geschlechterstereotype ist insbesondere in der Sozial- und Kognitionspsychologie verankert und bezeichnet kognitive Zuschreibungen von Eigenschaften als „weiblich“ und „männlich“. Es hatte in den 1970er und 1980er Jahren Konjunktur, was vermutlich mit dem Vorteil erklärt werden kann, dass es in hohem Maß mit empirischer Evidenz einhergeht, sowohl auf der Ebene alltagsweltlicher Empirie als auch in der empirischen Forschung.

7 Beispiele für diese Strategie gibt es viele, sowohl auf der Ebene der Medien, Materialien und Spielsachen, mit denen Kinder aufwachsen, die nun geschlechterbewusst gestaltet werden, als auch Erziehungspraktiken, die Kinder zur Ausbildung von Interessen und Fähigkeiten anregen sollen, die typischerweise dem, anderen' Geschlecht zugerechnet werden, also Fußball und Technikbaukästen für Mädchen, Puppenspiele und Sticken für Jungen. In Kritik solcher Ansätze entwickelt sich bald eine geschlechterreflektierende Pädagogik (zum Überblick vgl. Rendtorff 2006). 
kehr der Geschlechterforschung vom Sozialisationsparadigma führt (vgl. Dausien 1999). Zwei wissenschaftliche Strömungen sind dabei einflussreich, die sich aus unterschiedlichen theoretischen Perspektiven heraus mit den Identitätsannahmen und Tendenzen der Reifizierung in der bisherigen Frauen- und Geschlechterforschung auseinandersetzen:

Zum einen gewinnen bereits in den 1980er Jahren diskutierte sozialkonstruktivistische und interaktionistische Ansätze an Bedeutung, die Geschlecht als soziale bzw. kulturelle Konstruktion begreifen und starke empirische Konzepte zu ihrer Rekonstruktion zur Verfügung stellen. Das Konzept des doing gender (West/Zimmerman 1987) ermöglicht es nicht nur, substanzialisierende Vorstellungen von Geschlecht, Geschlechtsidentität oder, weiblichen/männlichen Eigenschaften' zu kritisieren, sondern bietet eine theoretisch und methodologisch überzeugende Alternative an: Geschlecht wird als eine fortlaufende soziale Praxis verstanden, als etwas, was Akteur_innen in konkreten sozialen Situationen tun, und nicht als etwas, was sie sind oder haben (vgl. Garfinkel 1967; Goffman 1977; West/Zimmerman 1987; Lorber/Farrell (1991); zum Überblick vgl. Gildemeister 2010; Wetterer 2010).

Das doing gender-Konzept wird in den 1990er Jahren zunehmend in der deutschsprachigen Geschlechterforschung rezipiert (vgl. Hagemann-White 1984, 1988; Gildemeister/Wetterer 1992), in ethnographischen Studien erprobt (z.B. Breidenstein/Kelle 1998; Kelle 1999) und auch explizit gegen Grundannahmen der Sozialisationsforschung gewendet (vgl. Kelle/Breidenstein 1996). Der damit angeregte Perspektivwechsel ist radikal und irritierend, besonders für eine Frauenforschung, die sich in Kritik an der androzentrischen Wissenschaft auf positive Konzepte ,weiblicher' Forschung und Identität bezieht und bestrebt ist, damit eine eigenständige Position in der Wissenschaft zu fundieren. Darüber hinaus bricht der Ansatz radikal mit der Vorstellung, gender sei eine soziale Überformung eines doch irgendwie vorhandenen biologischen Kerns von Geschlecht (sex) - eine durchaus verbreitete Sichtweise, die ein Nebeneinander der bisherigen Auffassung und feministischer Kritik noch zugelassen hatte.

Es wundert somit nicht, dass doing gender in pädagogischen Kontexten kontrovers diskutiert wurde und wird. Andererseits bestärkte der Ansatz auch handlungsorientierte Perspektiven und die Idee, dass Individuen nicht nur Opfer gesellschaftlicher Verhältnisse sind, sondern auch „Täter_innen“ (Thürmer-Rohr 1983) - und dass sie deshalb auch aktiv an der Veränderung gesellschaftlicher Verhältnisse mitwirken können, z.B. im Rahmen einer pädagogischen Praxis, die sich als geschlechterreflexiv begreift und ihre eigene Beteiligung an Prozessen des doing gender in den Blick nimmt.

Eine zweite Richtung, die sich kritisch mit der bisherigen Frauen- und Geschlechterforschung auseinandersetzt und ein mindestens ebenso hohes Irritationspotenzial enthält, ist mit dem Begriff der Dekonstruktion verbunden. Im Anschluss an die französische poststrukturalistische Philosophie, insbesondere 
an die Konzepte Jacques Derridas sowie an Arbeiten von Judith Butler und Michel Foucault, kritisiert sie die Idee des autonomen Subjekts und Identitätskonzepte in der Geschlechterforschung. Das 1991 ins Deutsche übersetzte Buch „Gender Trouble“ (Butler 1990) bringt ein verschiedentlich schon formuliertes ,Unbehagen“ mit der Theorie und Praxis der Geschlechterforschung auf den Punkt. Es wird zum Kristallisationskern einer Debatte (vgl. Benhabib u.a. 1993), die weit über den Feminismus hinaus wirkt und allmählich auch die erziehungswissenschaftliche Diskussion erreicht, wo Ansätze der Queer Theory (Hark 1996) auch mit pädagogischen Fragen verbunden werden (Plößer 2005).

Die dekonstruktivistische Debatte setzt sich zwar nicht direkt mit dem Sozialisationsparadigma auseinander, bewirkt aber eine Perspektivenverschiebung in Richtung der gesellschaftlichen Diskurse und kulturellen Ordnungen, die geschlechtliche Subjekte machtvoll hervorbringen. Vorrangiges Ziel ist die Dekonstruktion kultureller Denksysteme, verbunden mit einem zunächst eher theoretischen und politischen, weniger empirisch-forschenden Interesse an irritierenden und subversiven Praktiken (Butler 1990). Ansätze, die dekonstruktivistische Theorie mit Fragen der Sozialisation zu verbinden und im Rahmen empirischer Studien zu untersuchen, werden erst später entwickelt (vgl. dazu Hartmann in diesem Band).

Beide Richtungen, die sozialkonstruktivistische und die dekonstruktivistische, unterscheiden sich in ihren theoretischen Begriffen und Denktraditionen, dennoch tragen sie, zumal in ihrem etwa zeitgleichen Auftreten, dazu bei, dass das Sozialisationsthema aus dem Blick gerät und vermeintlich obsolet wird ${ }^{8}$ (vgl. Dausien 1999). Die Rede von Geschlechtersozialisation oder gar geschlechtsspezifischer Sozialisation gerät in Verdacht, jener kritisierten Idee eines relativ stabilen, ,weiblichen' oder ,männlichen' Subjekts noch immer anzuhängen. Die sozialisationstheoretische Sprache bekommt den Geschmack des Gestrigen, Altbackenen. Sie ,überwintert` jedoch in Lehr- und Handbüchern bzw. deren Neuauflagen.

Diese Entwicklung hat, rückblickend gesehen, nicht unproblematische Folgen: Zum einen wird das Forschungsfeld in der kritischen Auseinandersetzung homogenisiert; der Vorwurf eines substanzialisierenden und dichotomisierenden Denkens wird pauschal gegenüber Sozialisationskonzepten formuliert, auch da wo er unberechtigt ist. Damit werden Ansätze, die sich aus nicht-dualistischen Theorietraditionen (Pragmatismus, Marxismus, einigen Spielarten der Psychoanalyse) heraus mit Sozialisation befassen, in gewisser Weise vergessen oder zumindest im feministischen Diskurs nicht weiterentwickelt. Unterstützt wird diese Tendenz womöglich dadurch, dass die Literatur aus den

8 Davon erstaunlich unberührt sind allerdings Forschungen, die im herkömmlichen Sinn Geschlechtersozialisation mit Hilfe von Variablendesigns zu erfassen suchen, besonders in der Psychologie. Diese werden allerdings in der Geschlechterforschung ebenso wenig rezipiert, wie sie ihrerseits die feministische Erkenntniskritik zu Kenntnis genommen haben. 
1970er und 1980er Jahren in aktuellen Lehr- und Forschungskontexten der Erziehungswissenschaft nur noch selten rezipiert wird. ${ }^{9}$

Ein weiteres Problem besteht darin, dass mit der Kritik nicht nur sozialisationstheoretische Erklärungsansätze, sondern auch die ihnen zugrundeliegenden Fragen aus dem Blick geraten sind (vgl. Dausien 1999): die Frage nach dem Werden und Gewordensein konkreter gesellschaftlicher Individuen, d.h. nach der Konstruktion und Konstitution von Subjektstrukturen, und die damit verknüpfte Frage nach der Vergesellschaftungslogik, also den gesellschaftlichen Strukturen bzw. Strukturierungsprozessen, die spezifische Individualitätsformen und Integrationsmechanismen hervorbringen.

Die erste Frage hatte Gudrun-Axeli Knapp bereits in den 1990er Jahren seinerzeit in Kritik der doing gender-Ansätze - systematisch wieder auf die Tagesordnung gesetzt: „Wie kann man ,Gewordensein“ von Personen unter dem Gesichtspunkt, Geschlecht' fassen, ohne zu substantialisieren oder zu naturalisieren, aber auch: ohne in Rhetoriken zu verfallen, in denen die Dimension der biographischen Haftung von Erfahrungen, die subjektkonstitutive Verbindlichkeit von Individuation und Sozialisation, völlig aufgelöst ist?““ (Knapp 1997: 503). Wenig später kritisiert auch Andrea Maihofer die „Tabuisierung“ dieser Frage und plädiert ,für eine Wiederaufnahme einer sozialisationstheoretischen Perspektive“" (Maihofer 2002: 17), von der sie sich Aufschluss über den „Prozeß der Aneignung, im“ Individuum“ (ebd.: 15) verspricht. Eine erneute Diskussion dieser theoretischen ,Lücke" hat zwar gezeigt, dass es durchaus neuere Beiträge zum Thema gibt; diese beziehen sich aber weniger auf das Sozialisationsparadigma und seine Weiterentwicklung als auf Biographieforschung, pädagogische und psychoanalytische Subjekttheorien oder performanztheoretische Ansätze (vgl. Dausien 2002; Tervooren 2006; sowie die Beiträge in Bilden/Dausien 2006).

Im Vergleich zur Subjektproblematik hat die Frage nach den gesellschaftlichen Verhältnissen bzw. den Vergesellschaftungsstrukturen in der erziehungswissenschaftlichen Geschlechterforschung weniger Resonanz gefunden (vgl. dazu Bilden 2006; Dausien 2006). Sie scheint tatsächlich weitgehend aus dem Blick geraten zu sein. Wie kann dieser Verlust oder, weniger dramatisch ausgedrückt, das aufkommende Desinteresse an gesellschaftsanalytischen Perspektiven erklärt werden? Unsere These ist, dass die beiden für die feministische Theoriedebatte der 1990er Jahre wichtigen Strömungen des Sozialkonstruktivismus und der Dekonstruktion zwar zu berechtigter Kritik an der Sozialisationsforschung geführt haben, dass sie alleine jedoch nicht für deren Bedeutungsverlust verantwortlich waren. Hinzu kommt die enorme Wirkung eines Paradigmas, das seit den 1980er Jahren in den Sozialwissenschaften diskutiert wird und sich sehr schnell zum dominanten Deutungsmuster entfalten konnte: das Individualisierungstheorem.

9 Dies hat womöglich auch mit dem technischen Umstand zu tun, dass die meisten dieser Texte weder neu aufgelegt wurden noch digitalisiert vorliegen. 


\section{Individualisierung ohne Vergesellschaftung? Erklärungsversuch für den Bedeutungsverlust des Sozialisationsparadigmas}

Anfang der 1980er Jahre wurde eine soziologische Gesellschaftsdiagnose vorgelegt, die den Zeitgeist genau getroffen und seitdem den sozial- und erziehungswissenschaftlichen Diskurs massiv geprägt hat, sich aber auch in anderen gesellschaftlichen Feldern und Diskursen fest verankern konnte. Unter den Überschriften ,Individualisierung' (Beck 1983, 1986; Beck/Beck-Gernsheim 1994, 2002) und ,reflexive Moderne‘ (Beck/Giddens/Lash 1996) hat sich ein Erklärungsansatz etabliert, der die Auflösung oder zumindest Relativierung herkömmlicher sozialer Bindungen an Klassen- und Geschlechterlagen postuliert und den Blick auf die Vervielfältigung von Lebenslagen, die abnehmende Bindungskraft zentraler Institutionen und Deutungsmuster und auf die Individualisierung der Lebensführung lenkt.

Wurde diese Interpretation zunächst durchaus als kritische Beschreibungsfolie entwickelt, die den Wirkmechanismen einer neuen, reflexiven Moderne auf der Spur und in gewisser Weise ihrer Zeit prognostisch voraus war, so wurde sie mit dem Zusammenbruch der politischen und gesellschaftlichen Systemlogik (Kapitalismus vs. Sozialismus) von der historischen Entwicklung gewissermaßen eingeholt und zunehmend in ein affirmatives Deutungsmuster verwandelt. Mit der Durchsetzung neoliberaler Steuerungsstrukturen konnte sich die Individualisierungsthese nicht nur in der Sozialwissenschaft, sondern auch als gelebte Alltagspraxis weiter ausbreiten und stabilisieren.

Ein Kernpunkt dieser Analysen ist bis heute die These, dass das am Arbeitsmarkt und an der (männlichen, bürgerlichen) Berufs- und Bildungsbiographie orientierte Konzept des Lebenslaufs seine Plausibilität und Orientierungskraft verloren hat. Individuen haben deshalb die Chance und sind zugleich gezwungen, so die Argumentation, ihre biographischen Entwürfe, Strategien und Praxen der alltäglichen Lebensführung in einem weitaus größeren Ausmaß als vorhergehende Generationen , selbst' zu gestalten, immer wieder neu zu erfinden, aus eigener Kraft, Phantasie und Erfahrung heraus zu ,konstruieren'. Dabei können sie sich, so der Tenor der individualisierungstheoretischen Argumentation, nicht mehr auf eine fraglos akzeptierte ,Normalbiographie ' berufen (vgl. Kohli 1985). Dieser Umstand bedeutet mit Blick auf gesellschaftlich festgeschriebene Geschlechterpositionen Verunsicherung, aber auch Befreiung aus den Fesseln der , traditionellen geschlechtsspezifischen Sozialisation “ für Frauen - und für Männer.

Dieser Wandel vollzieht sich nicht nur auf der normativen Ebene, sondern betrifft auch die faktischen, statistisch beobachtbaren Strukturen des Lebenslaufs: Institutionelle Vorgaben im Bildungs- und Erwerbssystem haben an Ver- 
bindlichkeit verloren bzw. sind ,flexibilisiert ${ }^{\star}$ worden. Das sog. Normalarbeitsverhältnis mitteleuropäischer kapitalistischer Gesellschaften ist in Auflösung begriffen, neue Formen der Arbeit, der Kapitalakkumulation und der Existenzsicherung, über nationale Grenzen hinweg, gewinnen an Bedeutung und produzieren neue Bruchlinien sozialer Ungleichheit (Stichwort Intersektionalität; vgl. Walgenbach 2014).

Anders gesagt: Die Geländer individueller Lebensplanung und Lebensführung scheinen zunehmend fragil geworden oder ganz weggebrochen zu sein. Die Zahl möglicher Lebenswege hat sich vervielfacht. Zugleich tritt das Risiko, die ,falsche' Entscheidung zu treffen und die Verantwortung dafür am Ende sich selbst zuschreiben zu müssen, als historisch neue Norm und Erfahrung individueller Existenz in Erscheinung. Subjekte reagieren darauf, so die Fortführung des Arguments, mit einem gesteigerten Maß an Reflexion und „Biographisierung“" (Kohli 1985). Sie ,basteln“-im Rahmen ihrer je eigenen Erfahrungs- und Deutungshorizonte - ihre biographischen Entwürfe und überarbeiten sie immer wieder neu.

Soweit eine kurze Rekapitulation dieser geläufigen Denkfigur. Sie wird immer wieder herangezogen, um zu erklären, dass sich Geschlechterverhältnisse verändert und vermeintlich an Bedeutung verloren haben - gerade im Kontext des Bildungssystems. Das individualisierte Subjekt scheint nicht mehr auf bestimmte weibliche oder männliche Rollenmuster festgelegt, sondern hat Spielräume, die eigene Biographie zu gestalten und dabei auch sein individuelles Konzept von ,Frausein` oder ,Mannsein` zu entwerfen und zu erproben - mehr Spielräume, als ihm oft lieb ist.

\subsection{Individualisierung statt Sozialisation?}

In der Erziehungswissenschaft wie in der pädagogischen Praxis und ihren Institutionen ist diese These, angekommen'. Sie dient der Erklärung von Veränderungen in allen gesellschaftlichen Bereichen, die ehemals klassische Themen der geschlechterbezogenen Sozialisationsforschung waren: Die Familienformen haben sich diversifiziert (Stichworte: Patchworkfamilien, Einelternfamilien), gleichgeschlechtliche Partnerschaften werden zunehmend legalisiert und als Erziehungskontexte erforscht, sexuelle Orientierungen jenseits des heteronormativen Modells werden als mögliche und legitime Optionen von Heranwachsenden ernst genommen. Auch gleichgeschlechtliche Elternschaft ist nicht nur denkbar, sondern taucht als reale Praxis in pädagogischen Institutionen auf.

Und mehr noch: Das heteronormative, streng dichotomisch strukturierte Geschlechtermodell selbst scheint langsam gelockert zu werden. Die Möglichkeit eines Geschlechtswechsels oder sogar einer anerkennungsfähigen Biographie, die sich ,zwischen“ den Geschlechtern positioniert, wäre noch vor einer 
Generation unaussprechlich, ja undenkbar gewesen. Inzwischen gibt es Literatur, Berichte in den Medien, sozial- und erziehungswissenschaftliche Forschungen (Gregor 2015) und erste Ansätze, die Themen Trans- und Intersexualität in die Ausbildung von Pädagog innen zu integrieren. Leitidee ist dabei, Kinder und Jugendliche pädagogisch zu begleiten, da diese nun selbst - und nicht mehr institutionelle Zwänge - darüber entscheiden sollen, welche Identität sie ausbilden und mit welchem Körper sie leben wollen.

Auch die Perspektive auf Bildungs- und Berufswege hat sich geändert. Mädchen stehen nicht nur formal alle Bildungsabschlüsse offen, sie erreichen sie auch. Zumindest oberflächlich betrachtet gibt es einen breiten Konsens, dass Jugendliche in ihrer Berufswahl nicht mehr an Geschlechtergrenzen scheitern sollten. Selbst Arkanbereiche der Männlichkeit wie Polizei und Militär sind inzwischen realistische Berufsoptionen für Frauen geworden, unterstützt durch mediale Vorbilder wie toughe Fernsehkommissarinnen, die zugleich auch noch sexy und alleinerziehende Mütter sind. Andere Berufe durchlaufen (weithin unbemerkt) einen Geschlechtswechsel, wie z.B. die Veterinärmedizin. Aber da sind auch Gegenbeispiele: Die sog. MINT-Fächer bleiben offensichtlich eine Bastion der Männlichkeit, Frühpädagogik und Pflege sind nach wie vor, weiblich'. Die Frage, wie das Bildungssystem auf gesellschaftliche Partizipation und ein Berufsleben vorbereitet (und welche pädagogischen Praxen sich daraus ableiten lassen), wird insgesamt gesehen jedoch kaum noch mit Blick auf Geschlecht diskutiert - und dies ist, gemessen an der jahrhundertelangen geschlechterdifferenzierenden (und -spaltenden) Tradition der Pädagogik, ein wirkliches Novum.

Stattdessen dominiert in der Pädagogik wie in der Bildungspolitik die Leitidee des ,sich selbst bildenden Individuums', das sich über die gesamte Lebensspanne hinweg, zumindest solange die körperlichen und geistigen Kräfte reichen, selbst organisiert, selbst managt, ja auch selbst sozialisiert ${ }^{10}$ und in eine Zukunft hinein entwirft, für die es keine Vorbilder in den vorangegangenen Generationen mehr gibt. Diese Idee hat neue Konzepte auf die Tagesordnung gesetzt, ,lebenslanges Lernen“, ,Übergänge“ (transitions) und ,Bildungsbiographien', die sowohl die empirische Bildungsforschung als auch pädagogische Theoriediskurse in großem Stil beschäftigen und z.T. neu ausrichten. Sie prägen auch neue Verständnisse pädagogischen Handelns, die ihren Kern in der Begleitung, Beratung und Moderation individueller Bildungswege haben (vgl. Dausien 2011a, b).

Welche Rolle spielt nun in diesen Ansätzen das Thema Sozialisation von Geschlecht? Einfach gesagt: es kommt kaum noch vor. Seit Mädchen und Frauen im Bildungssystem, gleichgezogen' oder ihre männlichen Genossen sogar ,überholt ${ }^{\star}$ haben, scheint es keinen Bedarf mehr für das Konzept der geschlechtsspezifischen Sozialisation zu geben. Lediglich da, wo Ungleichheiten

10 Vgl. dazu das Themenheft der Zeitschrift für Soziologie der Erziehung und Sozialisation, Heft 2/2002. 
zwischen den Geschlechtern hartnäckig an der empirischen Oberfläche oder in der pädagogischen Praxis auftauchen, wird noch auf Sozialisation als Erklärung zurückgegriffen. Beispiele hierfür sind das schon genannte Thema ,Mädchen und MINT-Fächer' oder der Diskurs um die Benachteiligung von Jungen im (feminisierten) Schulsystem (siehe dazu auch Bereswill/Ehlert in diesem Band).

Und es gibt ein weiteres Feld, in dem systematisch auf die Erklärung, Sozialisation' zurückgegriffen wird: nämlich die Thematisierung der Bildungswege von Jugendlichen ,mit Migrationshintergrund'. Ihnen (oder ihren Eltern) wird, zugespitzt gesagt, der Status des individualisierten Subjekts noch nicht in vollem Umfang zuerkannt, ihnen wird unterstellt, sie seien ,noch' den traditionellen Geschlechterrollen ihrer ,Kultur' verhaftet. Das Sozialisationsargument wird in solchen Kontexten mit der Idee der Rückständigkeit assoziiert: bestimmte Berufsfelder oder Lebensentwürfe, vor allem aber die Subjekte, die sich an diesen orientieren, ,sind noch nicht so weit', dass sie die Geschlechterfrage individuell handhaben und souverän hinter sich lassen könnten.

Diese Beobachtungen an der Oberfläche des pädagogischen Diskurses zusammenfassend, ziehen wir als Fazit: Das Individualisierungsparadigma, das sich auch, ja sogar wesentlich, auf Transformationen im Geschlechterverhältnis bezieht, hat das Thema Sozialisation abgelöst. Das Konzept, geschlechtsspezifische Sozialisation" erscheint überholt und bis auf weiteres im Übrigen auch kaum noch dazu brauchbar, sich wissenschaftlich zu profilieren. Die Rezeption des Individualisierungskonzepts erfolgt jedoch verkürzt. Während Analysen, die sich auf Individualisierungsprozesse der Subjekte und ihrer Lebensweisen beziehen, rasch in die erziehungswissenschaftliche Debatte übernommen werden, bleiben gesellschaftsbezogene Analysen weitgehend unbeachtet. Zugespitzt gesagt: Individualisierung wird als Theorem genutzt, ohne die Frage nach Vergesellschaftung zu stellen.

\subsection{Kritik am Individualisierungstheorem: Neue Formen der Subjektivierung und ,individualisierte Weiblichkeit“}

Die beschriebene These der Individualisierung wird in der Bildungswissenschaft nicht nur affirmativ aufgegriffen, sondern auch kritisch analysiert, insbesondere aus subjektivierungstheoretischer Sicht im Anschluss an Foucaults Arbeiten zu Disziplinierung und Gouvernementalität. Ein Beispiel dafür ist die kritisch-dekonstruktivistische Auseinandersetzung mit dem Diskurs des lebenslangen Lernens (z.B. Rothe 2011). Hier konnte überzeugend herausgearbeitet werden, wie im neoliberalen Diskurs (auch und gerade im erziehungswissenschaftlichen Feld) gesellschaftliche Strukturprobleme in individuelle Lernprobleme verwandelt und Individuen in die Subjektposition des/der Ler- 


\section{Bettina Dausien, Katharina Walgenbach}

nenden gebracht werden, und zwar als individuelle Lernende, die auch individuell für die Ergebnisse ihres Lernens und deren Verwertung Verantwortung tragen.

Diese Kritik lässt sich mit Christine Thons Analysen zum Individualisierungsdiskurs verknüpfen. Unter Bezugnahme auf Laclau und Mouffe formuliert sie die These, dass ,,individualisierte Weiblichkeit" als hegemoniales Subjektmodell des Neoliberalismus zu betrachten sei (Thon 2012). In Auseinandersetzung mit modernisierungstheoretischen Ansätzen und Diagnosen zeitgenössischer Transformationen im Geschlechterverhältnis interpretiert sie in Anknüpfung an Arbeiten von Angelika Wetterer (2003) und Angela McRobbie (2010) Individualisierung als „Form der herrschaftsförmigen Subjektivierung, die Subjekte als Individuen hervorbringt und sie in ein spezifisches Verhältnis zum Sozialen setzt und insofern auch für die Stabilisierung von Geschlechterordnungen relevant ist“" (Thon 2012: 33). Und weiter: „Individualisierte Weiblichkeit ist eine Formation, die das jeweilige Subjekt unter den gegenwärtigen kulturellen und ökonomischen Bedingungen sozial integrierbar und handlungsfähig macht" (ebd:: 36).

Dieses veränderte Verhältnis zum Sozialen, das sowohl in den apologetischen Ansätzen der Individualisierungsdebatte als auch in den kritischen subjektivierungstheoretischen Analysen in den Blick genommen wird, besteht in einer Akzentuierung von Individualität, deren strukturelle gesellschaftliche Bedingungen undeutlich geworden sind, unter diversen Schichten von Diskursen und Gegendiskursen verborgen.

Mag dies als Beschreibung alltagsweltlicher Verhältnisse zutreffen, so kann es als wissenschaftliche Analyse doch nicht genügen. Wir plädieren dafür, einen genaueren Blick auf die gesellschaftlichen Strukturen ,hinter ${ }^{6}$ den individualisierenden Diskursen in der Pädagogik in die erziehungswissenschaftliche Geschlechterdebatte wieder einzuführen (vgl. dazu Nickel 2012 sowie weitere Beiträge in Moser/Rendtorff 2012). Es geht darum, sich nicht mit Oberflächenbeschreibungen zu begnügen, die dem Individualisierungsdiskurs aufsitzen, sondern - so unsere These - die theoretisch-analytischen Potenziale des Sozialisationsparadigmas wieder in Erinnerung zu rufen.

Als Zwischenbilanz lässt sich festhalten, dass die Theoriekonjunkturen in der Erziehungswissenschaft bzw. der erziehungswissenschaftlichen Sozialisationsforschung - so gegensätzlich sie auch gewesen sein mögen - in ihrem Zusammenwirken den Blick auf gesellschaftliche Transformationsprozesse von Geschlechterverhältnissen geradezu verstellt haben. Durch die Verlagerung des erziehungswissenschaftlichen Interesses auf die Analyse von Mikropraktiken und -diskursen sowie auf Prozesse der Individualisierung wurden Sozialisationsperspektiven in den letzten Jahrzehnten marginalisiert. Wir möchten hingegen für eine Revitalisierung der sozialisationstheoretischen Perspektive in der erziehungswissenschaftlichen Geschlechterforschung plädieren. 
Die Wiederaufnahme gesellschaftsanalytischer Perspektiven erscheint uns nicht zuletzt aufgrund aktueller ökonomischer, politischer und sozialer Entwicklungstrends dringend geboten.

\section{Teil II}

\section{Wiederaufnahme gesellschaftsanalytischer Perspektiven in der Sozialisations- und Geschlechterforschung}

Seit der Jahrtausendwende wird in den Sozialwissenschaften verstärkt diskutiert, inwiefern sich durch die Neuordnung von Ökonomie, Staat und Gesellschaft Konstellationen ausmachen lassen, die auch die Geschlechterverhältnisse beeinflussen bzw. durch diese entscheidend organisiert werden (vgl. Sauer 2001; Fraser 2009; Casale 2012; Walgenbach 2015). Diese Gesellschaftsanalysen verweisen unseres Erachtens auf tiefgreifende gesellschaftliche Transformationsprozesse. Eine zukünftige Sozialisationsforschung - und damit meinen wir nicht alleine Forschungen zur Geschlechtersozialisation muss sich deshalb der Herausforderung stellen, diese gesellschaftlichen Transformationsprozesse einzubeziehen. Sie werfen nicht nur für die empirische Forschung und Theoriebildung der Geschlechtersozialisation neue Fragen auf, sondern benötigen auch eine dezidiert sozialisationstheoretische Perspektive für ihre erziehungswissenschaftliche Bearbeitung.

Bevor wir diese These ausführen, soll zunächst skizziert werden, was wir unter der Neuordnung von Ökonomie, Staat und Privatsphäre verstehen. Dafür scheint uns ein Rekurs auf aktuelle Gesellschaftsdiagnosen in verschiedenen Disziplinen der Sozialwissenschaften lohnend. Im Anschluss daran möchten wir an ausgewählten Beispielen verdeutlichen, worin wir die Potenziale einer Revitalisierung gesellschaftsanalytischer Perspektiven in der Sozialisationsund Geschlechterforschung sehen.

Einschränkend soll darauf hingewiesen werden, dass wir im Folgenden Elemente der Veränderung stärker hervorheben als Momente der Kontinuität, die eigentlich einen gleichwertigen Stellenwert in der Analyse einnehmen müssten. Somit werden vor allem Tendenzen herausgearbeitet, die sicherlich nicht alle Arbeits-, Lebens- und Subjektivierungsformen durchdringen, allerdings zunehmend hegemonial zu werden scheinen und somit zumindest eine Orientierungsfunktion bzw. Abgrenzungsfolie für individuelle Lebensentwürfe herausbilden. 


\subsection{Aktuelle Gesellschaftsdiagnosen zur Neuordnung von Ökonomie, Staat und Privatsphäre ${ }^{11}$}

Nach Nancy Fraser befinden wir uns aktuell am Übergang zu einer neuen Gesellschaftsform, die sie als postfordistisch, transnational und neoliberal charakterisiert (Fraser 2009: 44). In der Konsequenz verliert eine fordistische Organisation von Ökonomie, Staat und Gesellschaft zunehmend an Bedeutung, die sich seit Anfang des 20. Jahrhunderts beispielsweise durch Massenproduktion, Massenbeschäftigung, Massenkonsum, staatliche Wachstumssteuerung und den Ausbau eines Wohlfahrtsstaats auszeichnete (Hirsch/Roth 1986). In der Geschlechterforschung wurde vielfach herausgearbeitet, dass diese Gesellschaftsformation primär auf männliche Normalarbeitsverhältnisse, Lebensformen und Biographien ausgerichtet war.

Darüber hinaus war die Trennung zwischen Privatheit und Öffentlichkeit ein zentrales Signum des fordistischen Genderregimes (vgl. Young 1998). Dieses manifestierte sich etwa im Idealbild der heterosexuellen Kleinfamilie als Sozialisationsinstanz, in der Frauen vorzugsweise die (unbezahlte) Reproduktionsarbeit übernehmen und Männer die (bezahlte) Produktionsarbeit. Auf diese Form der , geschlechtsspezifischen' Arbeitsteilung rekurrierten auch Sozialisationstheorien der 1980er Jahre zur Herausbildung differenter Geschlechtsidentitäten, was bereits in Teil I skizziert wurde. Beispiele dafür sind die Objektbeziehungstheorie von Nancy Chodorow (Chodorow 1985) oder Carol Gilligans Studie zur weiblichen Moralentwicklung (Gilligan 1988).

Regina Becker-Schmidt verwies dagegen mit ihrem Konzept der ,doppelten Vergesellschaftung' auf die Tatsache, dass solche Theorien zur ,geschlechtsspezifischen Sozialisation' einen starken Mittelschicht-Bias haben. Ihre Studie zu Fabrikarbeiterinnen aus den 1980er Jahren zeigte, dass die befragten Frauen über widersprüchlich strukturierte Praxisbereiche in soziale $\mathrm{Zu}$ sammenhänge eingebunden waren, die sie durch persönliche Anstrengungen rekombinieren mussten (Becker-Schmidt 1987).

Allerdings basierte auch das Konzept der ,doppelten Vergesellschaftung ${ }^{6}$ auf einer historischen Konstellation, die durch eine Trennung zwischen Erwerbssphäre und Privatsphäre gekennzeichnet war. Die historische Genese dieser geschlechtlich codierten Sphärentrennung hatte Karin Hausen bereits in den Anfängen der Geschlechterforschung instruktiv herausgearbeitet. Sie zeigte, wie die Dichotomie von Öffentlichkeit versus Privatheit die Geschlechterordnung in der europäischen Moderne in fundamentaler Weise begründet. Dies galt insbesondere für das letzte Drittel des 18. Jahrhunderts bis in die zweite Hälfte des 20. Jahrhunderts (Hausen 1976).

11 Eine ausführliche Untersuchung der gesellschaftlichen Transformationsprozesse mit umfassenden Literaturhinweisen findet sich bei Walgenbach 2015. Der Beitrag fokussiert allerdings keine Sozialisationsfragen. 
Nach Hausen strukturiert die neue Geschlechterordnung aber auch zeitgenössische Vorstellungen von Staat, Gesellschaft, Familie, Humanität sowie die „Harmonisierung der menschlichen Verhältnisse durch die Ergänzung von Welt und Heim“ (Hausen 1976: 380). Exemplarisch lässt sich hier auf Wilhelm von Humboldts Bildungstheorie verweisen, der von einem komplementären Verhältnis zwischen Bildung/Staat und Erziehung/Privatsphäre ausging, welches bei ihm auch geschlechtlich codiert war (Humboldt [1792] 2002; siehe auch Casale 2012: 131-134).

Mit anderen Autor innen in den Sozialwissenschaften gehen wir davon aus, dass sich gegenwärtig eine Neuordnung von Ökonomie, Staat und Privatsphäre beobachten lässt, welche die vergeschlechtlichte Sphärentrennung modifizieren könnte oder zumindest in eine Krise führt (Sauer 2001; Fraser 2009; Casale 2012). Unter dem Label ,Postfordismus' wird diskutiert, inwiefern sich seit den 1970er Jahren neue Produktions- und Arbeitsformen in verschiedenen Branchen herausbilden (Hirsch/Roth 1986). Geläufige Stichworte dafür sind etwa lean production oder lean management, aber auch Dezentralisierung, Teamarbeit oder, flache Hierarchien'. Die Kontingenz bzw. Dynamik des Marktes wird hier also zum zentralen Strukturierungsmoment betrieblicher Organisation (Sauer 2007).

Die neuen Arbeits- und Produktionsformen führen zu einer Erosion der Trennung zwischen Öffentlichkeit und Privatheit (Sennet 2000; Boltanski/ Chiapello 2006). Es entstehen neue Formen der, Subjektivierung von Arbeit ${ }^{\star}$ und der ,Entgrenzung von Arbeit', die dazu führen, dass in immer mehr Branchen und Beschäftigungsverhältnissen die Bereiche ,Arbeit' und ,Leben ' eine zeitliche und räumliche Flexibilisierung erfahren (Pongratz/Voß 2003; Jürgens 2006). Die geschlechtlich codierte Trennung zwischen Öffentlichkeit und Privatheit wird durch solche Entwicklungsdynamiken also herausgefordert. Für die Sozialisationsforschung wäre es darüber hinaus interessant zu untersuchen, inwiefern dieser Wandel der Arbeitsorganisation auch berufliche Sozialisationsprozesse verändert oder gar neue Formen der Subjektivierung im Sozialisationskontext Berufswelt hervorbringt.

Jenseits der Veränderungen im ökonomischen Feld erscheinen uns aber auch weitere Entwicklungstrends bedeutsam. So werden die beschriebenen Transformationsprozesse durch eine neoliberale Wirtschafts- und Sozialpolitik flankiert, deren Denkweise sich bis in die 1930er Jahre zurückverfolgen lässt und deren Einfluss spätestens seit dem Wegfall der Systemkonkurrenzen (Kapitalismus vs. Sozialismus) in Europa entscheidend zunimmt. Die Grundidee des Neoliberalismus basiert darauf, dass alles dem Gesetz des Wettbewerbs bzw. Marktes unterworfen werden kann und soll (Ptak 2004). Mit dieser neoliberalen Logik sehen sich seit einigen Jahren auch zentrale Sozialisationskontexte wie Bildungsinstitutionen konfrontiert. Ein Beispiel dafür sind Hochschul-Rankings oder international vergleichende Schulleistungstests, welche 


\section{Bettina Dausien, Katharina Walgenbach}

die Konkurrenz auf einem ,Bildungsmarkt ${ }^{`}$ artifiziell herstellen. Welche Bedeutung dies für die Herausbildung neuer ,Bildungssubjekte" hat, scheint uns noch weitgehend ein Forschungsdesiderat zu sein.

Wichtig sind unseres Erachtens auch Transformationsprozesse im Feld der Politik, wo sich eine Verschiebung vom ,fürsorgenden Wohlfahrtsstaat‘ zum ,nationalen Wettbewerbsstaat' beobachten lässt (Hirsch 1995). In Deutschland wird diese Transformation spätestens seit den 1990er Jahren durch eine neoliberale Rhetorik begleitet, die auf einen Abbau des Wohlfahrtsstaates abzielt, der als Wachstumsbremse und Hindernis individueller Leistungsbereitschaft diskreditiert wird. Stattdessen protegieren neoliberale Akteure die Idee eines ,schlanken Staates' (Ptak 2004). Diese Orientierung zeigt sich auch in dem sozialpolitischen Paradigmenwechsel von Welfare to Workfare (Schröder/ Blair 1999). Die pädagogischen Konsequenzen dieser Entwicklung werden gegenwärtig vor allem in der Sozialpädagogik diskutiert (Kessl/Otto 2009); ob und welche Bedeutung sie für Sozialisationsprozesse haben, ist eine offene Frage.

Im Rekurs auf Karin Hausen wäre demnach heute erneut zu fragen, wie die Transformation von Ökonomie, Staat und Gesellschaft mit einer Neukonfiguration der Geschlechterordnung einhergeht. Aus erziehungswissenschaftlicher Perspektive hat sich Rita Casale mit dieser Frage befasst. Sie argumentiert, dass vor allem die Vorstellung einer komplementären Ergänzung von Staat und Familie, die den Bildungstheorien des 18. und 19. Jahrhunderts zugrunde liegt, aktuell in eine Krise gerät. Diesen Prozess bringt die Autorin dezidiert mit einer Transformation der sozialen und symbolischen Ordnung der Geschlechter in Verbindung. Einen Zusammenhang sieht sie in Tendenzen der Verstaatlichung der Erziehung und der Entstaatlichung der Bildung. Während sich der Staat zunehmend aus der Hochschulbildung zurückzieht, so Casale, führt das Interesse an einer Optimierung von ,Humankapital' zu Tendenzen einer Verstaatlichung von Erziehung. Die zeigt sich beispielsweise an Debatten über Frühförderung oder über die Zuständigkeit des Staates in Erziehungsfragen:

„Man ist mit einem ambivalenten Prozess konfrontiert, der einerseits zu einer neuen Bestimmung des Vertrags zwischen den Geschlechtern und zu einem veränderten Verständnis von Elternschaft führen könnte, und der andererseits die Einmischung des Staates in eine Sphäre beinhaltet, für die sich der neuzeitlichen Erziehungs- und Bildungstheorie zufolge der Staat nicht zuständig hielt" (Casale 2012: 135).

Auch für die Erziehungswissenschaft stellen sich somit grundsätzliche Fragen, z.B. inwiefern die Transformationsprozesse von Geschlechterverhältnissen zu einer Neubestimmung pädagogischer Handlungsfelder, Bildungsinstitutionen und erziehungswissenschaftlicher Leitbegriffe wie Bildung, Erziehung und Sozialisation führt (Walgenbach/Stach 2015). 


\subsection{Sozialisation und Geschlechterverhältnisse im Transformationsprozess}

Die oben beschriebenen Gesellschaftsdiagnosen bewegen sich allerdings meist auf einer Makroebene. Für die Sozialisationsforschung stellt sich nun die Frage, wie sie mit Analyseperspektiven verknüpft werden können, die sich auf die Subjektgenese beziehen: Welche Anrufungen bringt das neue Geschlechterregime hervor und wie werden diese von den Subjekten bearbeitet? Diesen Fragen soll im Folgenden anhand von vier Beispielen nachgegangen werden.

\section{Neue Formen der Subjektwerdung}

Mit der zunehmenden Entgrenzung von Arbeit entstehen neue Formen der Subjektivierung, die gegenwärtig mit Begriffen wie ,unternehmerisches Selbst' (Bröckling 2007) oder ,Arbeitskraftunternehmer' (Pongratz/Voß 2003) belegt werden. ,Eigenverantwortung' und ,Selbstmanagement' sind Schlüsselbegriffe des zentralen Prinzips einer marktgerechten Selbstregulation (vgl. Bröckling 2007; Michalitsch 2008). Dies gilt zum einen für die neuen Arbeitsformen, die mit Versprechungen von Selbstverwirklichung, Zeitsouveränität und Selbstbestimmung einhergehen, zum anderen wird die Marktlogik aber auch in das Individuum selbst verlagert, z.B. wenn Subjekte angerufen werden, sich als ihr eigenes Produkt zu vermarkten. Diese Aufforderung gilt zu jeder Zeit, da die Grenzen zwischen Arbeit und Freizeit verstärkt oszillieren (vgl. Michalitsch 2006: 90-96; Geissler 2008).

Hier wird also ein genuines Thema der Sozialisationstheorie und -forschung angesprochen, nämlich die Frage der Subjektwerdung. Abhängig vom jeweiligen theoretischen Zugang gilt es für eine zukünftige Sozialisationsforschung, die oben skizzierten gesellschaftlichen Transformationsprozesse mit Analysen zur Subjektivierung, Habitualisierung, Identitätsformationen, Subjektanrufungen oder Persönlichkeitsentwicklung zu verbinden. Nach Andrea Maihofer wäre allerdings gleichzeitig zu reflektieren, inwiefern die theoretischen Bemühungen, neue Identitätskonzepte zu beschreiben, selbst Effekt und/oder Motor gesellschaftlicher Veränderungen sind. Sie tragen somit zur Entstehung neuer ,Sozialcharaktere' oder ,Existenzmodi ' bei, die sich z.B. gegenwärtig durch Flexibilität, Mobilität oder die Fähigkeit zur Patchworkidentität auszeichnen (Maihofer 2002: 16).

In der Geschlechterforschung wird aktuell herausgearbeitet, dass Begriffe wie ,unternehmerisches Selbst' nicht allein auf eine Transformation der Erwerbssphäre hinweisen, sondern die Trennung zwischen Erwerbssphäre und Privatsphäre überschreiten. Das Private ist nicht länger nur politisch, resümiert Nina Power, es ist heute auch ökonomisch (Power 2011: 42). Christine Thon (2015) untersucht anhand von Broschüren, Handreichungen und Leitfäden von 


\section{Bettina Dausien, Katharina Walgenbach}

Ministerien, Stiftungen und Wirtschaftsverbänden, wie ökonomisierte Diskurse zur Vereinbarkeit von Familie und Beruf entsprechende ,Subjekte der Vereinbarkeit' selbst erst neu herstellen. Die Autorin arbeitet heraus, dass die Adressat innen dieser Publikationen (zumeist junge, gut ausgebildete Mütter) dazu angehalten werden, sich selbst als ,Humankapital' wahrzunehmen und den eigenen Marktwert als dringend gesuchte Fachkräfte zu erkennen.

Die Subjekte der Vereinbarkeit werden dabei, so Thon, als individualisierte Subjekte des Wollens, kompetenten Planens, strategischen Kommunizierens und ökonomisch rationalen, individuellen Problemlösens angerufen. Der pädagogische Impetus dieser Broschüren manifestiert sich beispielsweise in der Präsentation von Doppelkarrierepaaren als nachahmenswerten Vorbildern:

„Porträts von Eltern, die ,erfolgreiche“ Vereinbarkeitslösungen repräsentieren (vgl. BMFSFJ 2012b: 22f.; Baden-Württemberg-Stiftung 2010: 64ff.), sollen ein Lernen am Modell initiieren. Die Individualisierung von Vereinbarkeit erfolgt damit nicht nur über eine Responsibilisierung von Eltern (Kessl 2011: 69), sondern auch durch das Versprechen von Identitäten, die innerhalb des hegemonialen Diskurses mit einem hohen Maß an Attraktivität ausgestattet sind" (Thon 2015: 139).

Ausgespart bleiben hingegen geschlechtertheoretische Analysen, die das Problem der Vereinbarkeit auf einer institutionellen Basis bearbeiten und auf die widersprüchlichen Logiken von Erwerbsarbeit und Familienarbeit verweisen.

\section{Das Adult Worker Model als neuer Integrationsmodus}

Ein wichtiger Aspekt für die Wiederaufnahme gesellschaftsanalytischer Perspektiven in der Sozialisations- und Geschlechterforschung ist der aktuell diskutierte Paradigmenwechsel vom männlichen Familienernährermodell zum Adult Worker Model (vgl. Lewis 2001; Annesley 2007). Erklärungsfaktoren für die Etablierung des Adult Worker Model in Europa sind sowohl die bereits beschriebenen gesellschaftlichen Transformationsprozesse als auch ein prognostizierter demographischer Wandel. Befürchtet wird ein Fachkräftemangel, der in Europa verschiedene Wirtschaftsbranchen und Regionen in den nächsten Jahrzehnten unterschiedlich stark treffen wird (Walgenbach 2015: 31-32). Vergleichbar mit den 1960er Jahren werden vor diesem Hintergrund primär Frauen als ,Begabungsreserven' identifiziert, aber auch Migrant_innen und Jugendliche aus sogenannten ,bildungsfernen Schichten' (Walgenbach 2015: 32).

Junge, hochqualifizierte Frauen avancieren seit einigen Jahren zu wirtschaftspolitischen Hoffnungsträgerinnen, denn sie verfügen im Durchschnitt mittlerweile über ein höheres Bildungsniveau als ihre männlichen Altersgenossen (z.B. Vereinigung der Bayrischen Wirtschaft 2007: 134). Top Girls nennt McRobbie die neue weibliche Generation, die sie als hoch motivierte Leistungsträgerinnen des neoliberalen Umbaus identifiziert und denen die 
Tore ins Erwerbsleben und zur Konsumkultur weit geöffnet werden (vgl. McRobbie 2010). Was dieser Entwicklungstrend für die Forschungen zur Geschlechtersozialisation bedeutet, ist weitgehend unklar. Wir wissen erst wenig darüber, wie das Adult Worker Model bzw. die neuen neoliberalen Subjektanrufungen von Frauen und Männern (sowie weiteren Geschlechtern) tatsächlich bearbeitet werden; wie sie neue Selbstkonzepte, Identitätsentwürfe oder Denk-, Wahrnehmungs- und Handlungsschemata hervorbringen oder die geschlechtsbezogene Arbeitsteilung in der Familie neu konfigurieren.

Das Adult Worker Model offeriert aber auch einen neuen Modus gesellschaftlicher Integration. Es etabliert ein normatives Leitbild, nach dem alle erwerbsfähigen Erwachsenen auch erwerbstätig sein sollen. Sie haben nicht nur das Recht, sondern auch die Pflicht, ihren Lebensunterhalt durch Erwerbsarbeit zu sichern und den Staat zu entlasten (Klinger 2014). Insofern hat das Modell auch einen Zwangscharakter. Dieser ergibt sich allein schon ökonomisch, da die Abschaffung des männlichen Ernährermodells auch bedeutet, dass eine Person alleine heute häufig nicht mehr eine ganze Familie ernähren kann. Das Doppelverdienermodell geht demnach mit einer Absenkung des Lohnniveaus, einem sinkenden Lebensstandard, einem Anstieg der pro Haushalt geleisteten Arbeitsstunden und verschärften Doppelschichten einher (vgl. Fraser 2009: 51f.).

Gleichzeitig reaktiviert das Adult Worker Model allerdings auch ein altes Versprechen, das seit der französischen Revolution nie vollständig eingelöst wurde, nämlich die volle Integration in die Gesellschaft, unabhängig von Geschlecht, Migrationshintergrund, sexueller Orientierung etc. Die alte sozialisationstheoretische Frage nach der Integration in die Gesellschaft erhält somit eine neue Antwort: Nach der neoliberalen Logik findet soziale Integration ausschließlich auf der Basis von Verwertbarkeit und Leistungsfähigkeit statt. Das meritokratische Versprechen der Moderne erhält somit erneut Auftrieb.

In diesem Zusammenhang lassen sich auch seit der Jahrtausendwende in Europa verschiedene Initiativen beobachten, die auf einen Abbau von Diskriminierung abzielen. Allerdings adressieren das Allgemeine Gleichbehandlungsgesetz (2006) und die Richtlinien der Europäischen Union zum Antidiskriminierungsrecht primär die Erwerbssphäre. Gemäß dem Adult Worker Model sollen Barrieren für den Eintritt in den Arbeitsmarkt entfernt werden. Des Weiteren bleiben bestimmte Diskriminierungsmerkmale bzw. Ungleichheitsdimensionen wie etwa Schicht bzw. soziales Milieu oder Staatsangehörigkeit von der europäischen Antidiskriminierungspolitik explizit ausgeschlossen (vgl. Hormel 2008: 22).

Letztlich geht der Abbau von Diskriminierung mit einer gleichzeitigen Verschärfung von sozialer Ungleichheit einher (Walgenbach 2015). Die Individuen, die sich in die Sphäre der Produktion und Verwertbarkeit eingliedern lassen und den Staat von Transferleistungen entlasten, erfahren eine Integration als respektable Gesellschaftsmitglieder, während sich die Prozesse sozialer 
Spaltung für diejenigen verschärfen, die jenseits des Leistungskollektivs platziert sind. Für ihre gesellschaftliche, soziale und kulturelle Integration fühlt sich auch kein ,aktivierender Sozialstaat' mehr verantwortlich.

\section{Männliche Sozialisation im Transformationsprozess}

Der Strukturwandel der Erwerbsarbeit bedeutet für Männer eine Erosion des männlichen Normalarbeitsverhältnisses (Aulenbacher 2009). Fordistische Männlichkeitskonstruktionen assoziierten Männlichkeit primär mit Beruf und Karriere, wie auch diverse Studien zu männlichen Sozialisationsprozessen und Lebensentwürfen zeigen (z.B. Scholz 2004; Meuser 2006). Männer sind heute in einem neuen Ausmaß von Prekarität betroffen, während Frauen sich gerade durch den Strukturwandel neue Beschäftigungsperspektiven erschließen konnten (Lengersdorf/Meuser 2010). Nach Meuser geht Männern durch die Angleichung von ,männlichen' und ,weiblichen' Erwerbsverläufen gegenwärtig ein wichtiges „Distinktionsmittel“ verloren (Meuser 2010: 331).

Aktuelle Prozesse der neoliberalen Globalisierung motivierten Raewyn Connell, ihr Konzept der hegemonialen Männlichkeit neu zu überarbeiteten. Für Connell und Wood bildet die Transnational Business Masculinity heute eine neue Form hegemonialer Männlichkeit, deren Prototyp global agierende Top Manager sind. Ihre Orientierungen, Werte und Handlungsmuster setzen gegenwärtig auch komplizenhafte, untergeordnete und marginalisierte Formen von Männlichkeiten neu in Relation (vgl. Connell/Wood 2005; Connell 2010).

Es ist davon auszugehen, dass die gesellschaftlichen Entgrenzungsprozesse auch für die Eliten bzw. Repräsentanten hegemonialer Männlichkeit neue Herausforderungen darstellen, jene begegnen ihnen jedoch mit anderen Ressourcen und Bewältigungskompetenzen. Aufgrund besserer ökonomischer und sozialer Ausgangsbedingungen kann Unsicherheit hier eher aktiv gestaltend bewältigt werden (Meuser 2010: 332). Allerdings verstärken sich auch für Repräsentanten der hegemonialen Männlichkeit die persönlichen Risiken. Zum Beispiel stellt sich die Frage, ob zunächst positiv besetzte Attribute der Transnational Business Masculinity wie Flexibilität, Mobilität oder Autonomie auch mit Verlusten einhergehen, da sie z.B. mit engen sozialen (Familien-)Bindungen nicht vereinbar sind (Walgenbach 2015: 34).

Welche neuen männlichen Sozialisationsmuster sich in aktuellen Transformationsprozessen herausbilden, ist ebenfalls weitgehend ein Forschungsdesiderat. Erste Studien zu Männern in prekären Beschäftigungsverhältnissen weisen darauf hin, dass traditionelle Männlichkeitsmuster für Männer aus unteren sozialen Milieus nach wie vor eine zentrale Orientierung darstellen (z.B. Dörre 2007; Kreher 2007). Offenbar sind traditionell männliche Lebensentwürfe gerade für diejenigen Männer attraktiv, die sie kaum erreichen können (Bereswill 
2007). Einige Autor_innen verweisen zudem auf Momente der Neuaushandlung von Geschlechterarrangements, wenn Frauen beispielsweise ungeplant zu Familienernährerinnen werden (Völker 2009).

\section{Dethematisierung von Geschlechterhierarchien}

Ein letztes Beispiel für die Notwendigkeit der Wiederaufnahme gesellschaftsanalytischer Perspektiven in der Sozialisations- und Geschlechterforschung ist die These, dass gerade der jüngeren weiblichen Generation keine Sprache zur Verfügung zu stehen scheint, um die nach wie vor existierenden Geschlechterhierarchien zu kritisieren (vgl. dazu auch Klinger in diesem Band).

Koppetsch und Burkart (1999) kommen auf der Basis ihrer empirischen Studie Die Illusion der Emanzipation zu dem Ergebnis, dass eine weit verbreitete Gleichheitsrhetorik die Thematisierung bestehender Geschlechterungleichheiten geradezu verhindert. Sie weisen nach, dass tradierte Geschlechternormen in den meisten sozialen Milieus zwar weitgehend ihre Gültigkeit verloren haben, dennoch finden sich bei heterosexuellen Paaren (insbesondere mit Kindern) nach wie vor primär traditionelle Formen geschlechtlicher Arbeitsteilungen. Neu ist allerdings, dass diese nicht mehr als geschlechtlich strukturiertes Ungleichheitsverhältnis wahrgenommen werden. Wenn Frauen z.B. für die Kindererziehung aus dem Berufsleben aussetzen, dann wird dies von den befragten Paaren als Ergebnis individueller bzw. ökonomischer Abwägungen interpretiert (Koppetsch/Burkart 1999). Im Sinne einer neoliberalen Rhetorik werden Entscheidungen wie die Aufteilung der Elternzeit,gemeinsam' und ,im Team' entschieden. Sie kommen somit auch nicht als Effekt einer strukturellen Geschlechterhierarchie in den Blick.

Nach McRobbie wird jungen Frauen im neoliberalen Geschlechterregime ein neuer ,Geschlechtervertrag' angeboten: Für die vollständige Integration in den Erwerbsmarkt und die Konsumsphäre verzichten sie auf einen als überholt geltenden Feminismus. Im Gegensatz zu traditionellen Backlash-Debatten, so McRobbie, werden die Erfolge des Feminismus nun zwar anerkannt, doch seine Inhalte, Protestformen und Utopien werden als unzeitgemäß ausrangiert. McRobbie spricht hier von einer „Politik der Desartikulation“, da gerade durch die Formen der Anerkennung bzw. Inanspruchnahme etwaige Aufrufe zur Erneuerung des Feminismus bereits im Vorfeld diskreditiert würden. Die Dethematisierung von Geschlechterungleichheit ist für die Autorin demnach ein zentrales Merkmal des neoliberalen Geschlechterregimes (vgl. McRobbie 2010).

Was bedeuten diese empirischen Befunde und theoretischen Überlegungen für eine Sozialisationsforschung, die sich für soziale Ungleichheiten, Diskriminierungsformen und Subjektivierungsprozesse interessiert? Inwiefern ist es für junge Frauen als Interviewpartnerinnen beispielsweise möglich, subjektive 
Erfahrungen von Unterdrückung oder ,Opfersein` überhaupt zu artikulieren? Mit welchen methodischen Verfahren und Instrumenten kann eine Sozialisationsforschung auf diese Herausforderung reagieren?

\section{Teil III}

\section{Ausblick}

Die beiden Argumentationslinien, mit denen wir uns an das komplexe Thema herangewagt haben, haben gewiss manche Frage nur gestreift und vermutlich mehr Fragen aufgeworfen als beantwortet. Dies war durchaus unsere Absicht. Wir sehen angesichts der beschriebenen gesellschaftlichen Transformationsprozesse und ihrer enormen Bedeutung für die Geschlechterordnung(en), in und mit denen wir leben, die Notwendigkeit, uns in der erziehungswissenschaftlichen Geschlechterforschung näher mit den entsprechenden Analysen auseinanderzusetzen.

Gleichzeitig weisen die skizzierten Gesellschaftsanalysen selbst wiederum ein Forschungsdesiderat auf: die Frage nach der Verknüpfung von Subjektwerdung und Vergesellschaftung. Die meisten der aktuellen Studien zu gesellschaftlichen Transformationsprozessen arbeiten zwar die Anrufungen heraus, denen sich Subjekte heute ausgesetzt sehen, aber nicht deren konkrete Bearbeitung durch die Individuen. Inwiefern werden diese Anrufungen beispielsweise von den Individuen inkorporiert, abgelehnt oder modifiziert? Dieses Forschungsdesiderat veranlasst uns zu der Feststellung, dass die Diskussion zur Transformation von Geschlechterverhältnissen umgekehrt auch einer dezidiert sozialisationstheoretischen Perspektive bedarf.

Es geht folglich darum, die Transformation von Geschlechterverhältnissen im Rahmen der Fragestellungen und Traditionen unseres Faches, aber diesen auch überschreitend, zu verarbeiten. Das bedeutet vor allem, sie auf Prozesse der Subjektbildung zu beziehen oder, genauer, das Verhältnis von Subjektbildung und gesellschaftlichen Strukturen in den Blick zu nehmen. Dafür, so unser Plädoyer, ist es nützlich, die versickerte Diskussion um Geschlechtersozialisation und insbesondere soziologische Theorien der Vergesellschaftung wieder aufzunehmen - nicht weil sie die richtigen Antworten schon geliefert hätten, aber weil sie Fragen stellen, die nach wie vor geeignet erscheinen, um Prozesse der Geschlechterkonstruktion in individuellen Biographien und gesellschaftlichen Verhältnissen zu analysieren; und weil sie in der Beantwortung der Fragen bereits einige theoretische Klärungen geleistet haben. 
Dabei sollten allerdings Probleme des Sozialisationsansatzes, die in der Vergangenheit erkannt und systematisch reflektiert wurden (s. Teil I), nicht wiederholt werden. So sind vor allem Ansätze aufzugreifen, die in theoretischer Hinsicht eine dialektische Grundkonzeption verfolgen, die sich auch in der Analyse singulärer Phänomene (an historisch-konkreten Fällen) anwenden lässt, statt die Komplexität des Themas durch additives Aneinanderfügen von Teil-Theorien und alles umfassenden ,Ebenen' aufzulösen.

In empirischer Hinsicht sind reflexiv-rekonstruktive Methodologien gefragt, die danach suchen, jenen oft verdeckten, inneren Zusammenhang ${ }^{6}$ zwischen dem Individuellen und dem Sozialen am konkreten Material bzw. ,am Fall' zu rekonstruieren, anders gesagt, die das Gesellschaftliche ,im‘ Individuum und das Individuelle als Erscheinungsform des Gesellschaftlichen in den Blick nehmen. Das bedeutet, wir brauchen theoretisch eingebettete und reflektierte empirische Ansätze.

Hinzu kommt die Aufgabe, die Konstruktivität des Forschungsprozesses systematisch in Rechnung zu stellen, also die wissenschaftlichen und gesellschaftlichen Kontexte der eigenen Forschung zu reflektieren und Prozesse der Ko-Konstruktion, die sich daraus ergeben, in die Analyse kritisch mit einzubeziehen. Ansätze, die sich diesen Ansprüchen verpflichtet sehen, finden sich gegenwärtig vor allem in der Biographieforschung (Dausien 2008; Thon 2008), einer auf Institutionen und Praxen bezogenen Ethnographie (Breidenstein u.a. 2013), in diskursanalytischen Studien, die empirisch-rekonstruktiv arbeiten (s. noch einmal Hartmann in diesem Band) oder auch in neu entwickelten theoriegeleiteten Konzepten wie der Habitusanalyse (Bremer/TeiwesKügler 2013). Auch theoretisch reflektierte Kombinationen zwischen quantitativen und qualitativen Ansätzen bieten ein noch unausgeschöpftes Potenzial für die Bearbeitung sozialisationstheoretischer Fragestellungen.

Entscheidend ist, so unser Fazit aus dem Rückblick in die Geschichte der Sozialisationsforschung, den Anspruch eines allumfassenden Sozialisationsmodells zu vermeiden und sich dafür auf eine theoretisch genaue, empirisch gehaltvolle und materialbezogene Analyse begründet ausgewählter Phänomene zu konzentrieren. Der erhoffte Gewinn ist die Entwicklung neuer tragfähiger Begriffe und Konzepte, mit denen wir die Komplexität und (verdeckte) Strukturierung von Sozialisationsprozessen an konkreten Fallbeispielen gedanklich besser fassen können.

Das klingt nur vermeintlich bescheiden. Eine der größten Herausforderungen besteht schon darin, die richtigen Fragen zu stellen und gute Beispiele für ihre Bearbeitung auszuwählen. Es gilt somit, sozialisationstheoretische Fragestellungen ,kleinzuarbeiten" und in theoretisch und methodologisch reflektierte Forschung zu übersetzen. Eine Hilfe auf dem Weg dahin ist - besonders für erziehungswissenschaftliche Forscher innen, die es gewohnt sind, Individuen und ihren sozialen Praktiken in den Blick zu nehmen - eine kritische 
Auseinandersetzung mit vorliegenden Analysen zu aktuellen Prozessen der gesellschaftlichen Umstrukturierung. Unser Essay sollte - mit allen dem Genre geschuldeten Verkürzungen und Zuspitzungen - dazu anregen, diese Überlegungen auf- und in die je eigenen Forschungsfelder mitzunehmen.

\section{Literatur}

Annesley, Claire (2007): Lisbon and Social Europe: Towards a European 'Adult Worker Model' Welfare System. In: Journal of European Social Policy 17, 3, S. $195-205$.

Aulenbacher, Brigitte (2009): Die soziale Frage neu gestellt. Gesellschaftsanalysen der Prekarisierungs- und Geschlechterforschung. In: Castel, Robert / Dörre, Klaus (Hrsg): Prekarität, Abstieg, Ausgrenzung. Die soziale Frage am Beginn des 21. Jahrhunderts. Frankfurt a.M. / New York: Campus, S. 65-80.

Bauer, Ullrich (2011): Sozialisation und soziale Ungleichheit. Eine Hinführung. Wiesbaden: VS.

Beauvoir, Simone de (1949): Le deuxième Sexe. Paris: Gallimard.

Beauvoir, Simone de (1951): Das andere Geschlecht. Sitte und Sexus der Frau. Hamburg: Rowohlt.

Beck, Ulrich (1983): Jenseits von Stand und Klasse? Soziale Ungleichheiten, gesellschaftliche Individualisierungsprozesse und die Entstehung neuer sozialer Funktionen und Identitäten. In: Kreckel, Reinhard (Hrsg.): Soziale Ungleichheiten. Soziale Welt, Sonderband 2. Göttingen: Schwartz, S. 35-74.

Beck, Ulrich (1986): Risikogesellschaft. Auf dem Weg in eine andere Moderne. Frankfurt a. M.: Suhrkamp.

Beck, Ulrich / Beck-Gernsheim, Elisabeth (1994): Riskante Freiheiten: Individualisierung in modernen Gesellschaften. Frankfurt a. M.: Suhrkamp.

Beck, Ulrich / Beck-Gernsheim, Elisabeth (2002): Individualization: Institutionalized Individualism and its Social and Political Consequences. London: Sage Publication.

Beck, Ulrich / Giddens, Anthony / Lash, Scott (1996): Reflexive Modernisierung. Eine Kontroverse. Frankfurt a. M.: Suhrkamp.

Beck, Ulrich (2008): Jenseits von Klasse und Nation: Individualisierung und Transnationalisierung sozialer Ungleichheiten. In: Soziale Welt. Zeitschrift für sozialwissenschaftliche Forschung und Praxis. Heft 4; 59. Jg., S. 301-325.

Beck, Ulrich (2009): Macht und Gegenmacht im globalen Zeitalter. Frankfurt a. M.: Suhrkamp.

Becker-Schmidt, Regina (1987): Die doppelte Vergesellschaftung - die doppelte Unterdrückung: Besonderheiten der Frauenforschung in den Sozialwissenschaften. In: Unterkircher, Lilo / Wagner, Ina (Hrsg.): Die andere Hälfte der Gesellschaft. Wien: Verlag d. Österreichischen Gewerkschaftsbundes, S. 10-25.

Benhabib, Seyla / Butler, Judith / Cornell, Drucilla / Fraser, Nancy (1993): Der Streit um die Differenz. Feminismus und Postmoderne in der Gegenwart [kommentiert (D)]. Frankfurt a. M.: Fischer. 
Bereswill, Mechthild (2007): Sich auf eine Seite schlagen. Die Abwehr von Verletzungsoffenheit als gewaltsame Stabilisierung von Männlichkeit. In: Bereswill, Mechthild / Meuser, Michael / Scholz, Sylka (Hrsg.): Dimensionen der Kategorie Geschlecht: Der Fall Männlichkeit. Münster: Westfälisches Dampfboot, S. 101118.

Bilden, Helga (1980): Geschlechtsspezifische Sozialisation. In: Hurrelmann, Klaus / Ulich, Dieter (Hrsg.): Handbuch der Sozialisationsforschung. Weinheim / Basel: Beltz, S. 777-812.

Bilden, Helga (1991): Geschlechtsspezifische Sozialisation. In: Hurrelmann, Klaus / Ulich, Dieter (Hrsg.): Neues Handbuch der Sozialisationsforschung. 4., völlig neubearbeitete Auflage. Weinheim / Basel: Beltz, S. 279-301.

Bilden, Helga (2006): Sozialisation in der Dynamik von Geschlechter- und anderen Machtverhältnissen. In: Bilden, Helga / Dausien, Bettina (Hrsg.): Sozialisation und Geschlecht. A.a.O., S. 45-70.

Bilden, Helga / Dausien, Bettina (Hrsg.) (2006): Sozialisation und Geschlecht. Theoretische und methodologische Perspektiven. Opladen / Farmington Hills: Barbara Budrich.

Boltanski, Luc / Chiapello, Ève (2006): Der neue Geist des Kapitalismus. Konstanz: UVK.

Borst, Eva / Casale, Rita (Hrsg.) (2007): Ökonomien der Geschlechter. Jahrbuch Frauen- und Geschlechterforschung in der Erziehungswissenschaft, Bd. 3. Opladen: Barbara Budrich.

Bourdieu, Pierre (1987): Die feinen Unterschiede. Kritik der gesellschaftlichen Urteilskraft. Frankfurt a. M.: Suhrkamp.

Breidenstein, Georg / Kelle, Helga (1998): Geschlechteralltag in der Schulklasse. Ethnographische Studien zur Gleichaltrigenkultur. Weinheim / München: Juventa.

Breidenstein, Georg / Hirschauer, Stefan / Kalthoff, Herbert / Nieswand, Boris (2013): Ethnografie. Die Praxis der Feldforschung. Konstanz: UVK.

Bremer, Helmut / Teiwes-Kügler, Christel (2013): Habitusanalyse als Habitus-Hermeneutik. In: Zeitschrift für Qualitative Forschung, Heft 2/2013, S. 199-219.

Bröckling, Ulrich (2002): Das unternehmerische Selbst und seine Geschlechter. Gender-Konstruktionen in Erfolgsratgebern. In: Leviathan, 30, 2, S. 175-194.

Bröckling, Ulrich (2007): Das unternehmerische Selbst. Soziologie einer Subjektivierungsform, Frankfurt a. M.: Suhrkamp.

Butler, Judith (1990): Gender Trouble. Feminism and the subversion of identity. New York / London: Routledge.

Butler, Judith (1991): Das Unbehagen der Geschlechter. Frankfurt a. M.: Suhrkamp.

Casale, Rita (2012): Verstaatlichung der Erziehung und Entstaatlichung der Bildung. Anmerkungen zur Krise der Komplementarität von Staat und Familie. In: Aubry, Carla / Geiss, Michael / Magyar-Haas, Veronika / Miller, Damian (Hrsg.): Positionierungen. Zum Verhältnis von Wissenschaft, Pädagogik und Politik. Weinheim: Beltz Juventa, S. 128-139.

Casale, Rita / Forster, Edgar (Hrsg.) (2011): Ungleiche Geschlechtergleichheit. Geschlechtergleichheit und Theorien des Humankapitals. Jahrbuch Frauen- und Geschlechterforschung in der Erziehungswissenschaft, Bd. 7. Opladen: Barbara Budrich.

Chodorow, Nancy (1985): Das Erbe der Mütter. Psychoanalyse und Soziologie der Mütterlichkeit. München: Frauenoffensive. 


\section{Bettina Dausien, Katharina Walgenbach}

Connell, Raewyn / Wood, Julian (2005): Globalization and business masculinities. In: Menand Masculinities, 7, 4, S. 347-364.

Connell, Raewyn (2010): Im Innern des gläsernen Turms: Die Konstruktion von Männlichkeiten im Finanzkapital. In: Feministische Studien, 28, 1, S. 8-24.

Dausien, Bettina (1999): „Geschlechtsspezifische Sozialisation“ - Konstruktiv(istisch)e Gedanken zu Karriere und Kritik eines Konzepts. In: Dausien, Bettina / Herrmann, Martina / Oechsle, Mechtild / Schmerl, Christiane / Stein-Hilbers, Marlene (Hrsg.): Erkenntnisprojekt Geschlecht. Feministische Perspektiven verwandeln Wissenschaft. Opladen: Leske und Budrich, S. 216-249.

Dausien, Bettina (2002): „Biographie“ und/oder „Sozialisation“? Überlegungen zur paradigmatischen und methodischen Bedeutung von Biographie in der Sozialisationsforschung. In: Kraul, Margret / Marotzki, Winfried (Hrsg.): Biographische Arbeit. Perspektiven erziehungswissenschaftlicher Biographieforschung. Opladen: Leske und Budrich, S. 65-91.

Dausien, Bettina (2006): Geschlechterverhältnisse und ihre Subjekte. Zum Diskurs um Sozialisation und Geschlecht. In: Bilden, Helga / Dausien, Bettina (Hrsg.): Sozialisation und Geschlecht. A.a.O., S. 16-43.

Dausien, Bettina (2008): Biographieforschung. In: Becker, Ruth / Kortendiek, Beate (Hrsg.): Handbuch der Frauen- und Geschlechterforschung. Theorie, Methoden, Empirie. Wiesbaden: VS Verlag für Sozialwissenschaften, 2., erweiterte und aktualisierte Aufl., S. 354-367.

Dausien, Bettina (2011a): „Biographisches Lernen“ und „Biographizität“ - Überlegungen zu einer pädagogischen Idee und Praxis in der Erwachsenenbildung. In: Hessische Blätter für Volksbildung, 2/2011, S. 110-125.

Dausien, Bettina (2011b): „Das beratene Selbst“ - Anmerkungen zu Bildungsbiographien im gesellschaftlichen Wandel und Strategien ihrer professionellen Bearbeitung. In: Hammerer, Marika / Kanelutti, Erika / Melter, Ingeborg (Hrsg.): Zukunftsfeld Bildungs- und Berufsberatung. Neue Entwicklungen aus Wissenschaft und Praxis. Bielefeld: W. Bertelsmann, S. 21-40.

Dörre, Klaus (2007): Prekarisierung und Geschlecht. Ein Versuch über unsichere Beschäftigung und männliche Herrschaft in nachfordistischen Arbeitsgesellschaften. In: Aulenbacher, Brigitte / Funder, Maria / Jacobsen, Heike / Völker, Susanne (Hrsg.): Arbeit und Geschlecht im Umbruch der modernen Gesellschaft: Forschung im Dialog. Wiesbaden: VS, S. 285-302.

Durkheim, Emile (1977): Über die Teilung der sozialen Arbeit [1893]. Frankfurt a. M.: Suhrkamp.

Elias, Norbert (1976): Über den Prozess der Zivilisation. Soziogenetische und psychogenetische Untersuchungen, 2 Bde. Frankfurt a. M.: Suhrkamp.

Fraser, Nancy (2009): Feminismus, Kapitalismus und die List der Geschichte. In: Blätter für deutsche und internationale Politik, 8, S. 44-57.

Garfinkel, Harold (1967): Studies in Ethnomethodology. Englewood Cliffs: PrenticeHall.

Geissler, Birgit (2008): Zeitsouveränität: die paradoxe Suche nach Selbstbestimmung. In: Wagner, Gabriele / Hessinger, Philipp (Hrsg.): Ein neuer Geist des Kapitalismus? Paradoxien und Ambivalenzen der Netzwerkökonomie. Wiesbaden: VS, S. 257-277.

Gildemeister, Regine (2010): Doing Gender. Soziale Praktiken der Geschlechterunterscheidung. In: Becker, Ruth / Kortendiek, Beate (Hrsg.): Handbuch Frauen- und 
Geschlechterforschung. Theorie, Methoden, Empirie. 3., erweiterte und durchgesehene Aufl. Wiesbaden: VS Verlag für Sozialwissenschaften, S. 137-145.

Gildemeister, Regine / Wetterer, Angelika (1992): Wie Geschlechter gemacht werden. Die soziale Konstruktion der Zweigeschlechtlichkeit und ihre Reifizierung in der Frauenforschung. In: Knapp, Gudrun-Axeli / Wetterer, Angelika (Hrsg.), TraditionenBrüche. Entwicklungen feministischer Theorie. Freiburg i. Br.: Kore, S. 201254.

Gilligan, Carol (1988): Die andere Stimme. Lebenskonflikte und Moral der Frau. München / Zürich: Piper.

Goffman, Erving (1977): The Arrangement between the Sexes. In: Theory and Society, 4, 301-331.

Gregor, Anja (2015, im Druck): Constructing Intersex: Intergeschlechtlichkeit als soziale Kategorie. Bielefeld: transcript.

Hagemann-White, Carol (1984): Sozialisation: Weiblich - männlich? Opladen: Leske und Budrich.

Hagemann-White, Carol (1988): Wir werden nicht zweigeschlechtlich geboren ... In: Hagemann-White, Carol / Rerrich, Maria S. (Hrsg.): FrauenMännerBilder. Männer und Männlichkeit in der feministischen Diskussion. Bielefeld: AJZ, S. 224-235.

Hark, Sabine (1996): Deviante Subjekte. Die paradoxe Politik der Identität. Opladen: Leske und Budrich.

Hausen, Karin (1976): Die Polarisierung der „Geschlechtscharaktere“ im 19. Jh. Eine Spiegelung der Dissoziation von Erwerbs- und Familienleben. In: Conze, Werner (Hrsg.): Sozialgeschichte der Familie in der Neuzeit Europas. Stuttgart: Klett, S. 363-393.

Heintz, Bettina / Nadai, Eva (1998): Geschlecht und Kontext. De-Institutionalisierungsprozesse und geschlechtliche Differenzierung. In: Zeitschrift für Soziologie, 27, S. 75-93.

Hirsch, Joachim / Roth, Roland (1986): Das neue Gesicht des Kapitalismus: vom Fordismus zum Post-Fordismus. Hamburg: VSA.

Hirsch, Joachim (1995): Der nationale Wettbewerbsstaat. Staat, Demokratie und Politik im globalen Kapitalismus. Berlin / Amsterdam: ID Archiv.

Hormel, Ulrike (2008): Diversity und Diskriminierung. In: Sozial Extra. Soziale Arbeit und Diversity, 11/12, S. 20-23.

Humboldt, Wilhelm v. ([1792] 2002): Ideen zu einem Versuch, die Grenzen der Wirksamkeit des Staates zu bestimmen (1792). In: Humboldt, Wilhelm v.: Schriften zur Anthropologie und Geschichte. Werke (hrsg. v. A. Flitner / K. Giel). Darmstadt: Wissenschaftliche Buchgesellschaft, S. 56-233.

Hurrelmann, Klaus / Ulich, Dieter (Hrsg.) (1980): Handbuch der Sozialisationsforschung. Weinheim / Basel: Beltz.

Jürgens, Kerstin (2006): Arbeits- und Lebenskraft: Reproduktion als eigensinnige Grenzziehung. Wiesbaden: VS.

Kelle, Helga (1999): Geschlechterunterschiede oder Geschlechterunterscheidung? Methodologische Reflexion eines ethnographischen Forschungsprozesses. In: Dausien, Bettina / Herrmann, Martina / Oechsle, Mechtild / Schmerl, Christiane / SteinHilbers, Marlene (Hrsg.): Erkenntnisprojekt Geschlecht. A.a.O., S. 304-324.

Kelle, Helga / Breidenstein, Georg (1996): Kinder als Akteure: Ethnographische Ansätze in der Kindheitsforschung. In: Zeitschrift für Sozialisationsforschung und Erziehungssoziologie, 16 (1), S. 47-67. 


\section{Bettina Dausien, Katharina Walgenbach}

Kessl, Fabian / Otto, Hans-Uwe (2009): Soziale Arbeit ohne Wohlfahrtsstaat? Zeitdiagnosen, Problematisierungen und Perspektiven. Weinheim / München: Juventa.

Klinger, Cornelia (2014): Gender in troubled times: Zur Koinzidenz von Feminismus und Neoliberalismus. In: Fleig, Anne (Hrsg): Die Zukunft von Gender. Begriff und Zeitdiagnose. Frankfurt a.M.: Campus, S. 126-160.

Knapp, Gudrun-Axeli (1990): Zur widersprüchlichen Vergesellschaftung von Frauen. In: Hoff, Ernst-H. (Hrsg.): Die doppelte Sozialisation Erwachsener. Zum Verhältnis von beruflichem und privatem Lebensstrang. München: DJI, S. 17-52.

Knapp, Gudrun-Axeli (1997): Differenz und Dekonstruktion: Anmerkungen zum „Paradigmenwechsel“" in der Frauenforschung. In: Hradil, Stefan (Hrsg.): Differenz und Integration: Die Zukunft moderner Gesellschaften. Verhandlungen des 28. Kongresses der Deutschen Gesellschaft für Soziologie 1996 in Dresden. Frankfurt a. M. / New York: Campus, S. 497-513.

Kohli, Martin (1985): Die Institutionalisierung des Lebenslaufs. Historische Befunde und theoretische Argumente. In: Kölner Zeitschrift für Soziologie und Sozialpsychologie, 37, S. 1-29.

Koppetsch, Cornelia / Burkart, Günter (1999): Die Illusion der Emanzipation: zur Wirksamkeit latenter Geschlechtsnormen im Milieuvergleich. Konstanz: UVK.

Kreher, Thomas (2007): „Heutzutage muss man kämpfen“. Bewältigungsformen junger Männer angesichts entgrenzter Übergänge in Arbeit. Weinheim / München: Juventa.

Lengersdorf, Diana / Meuser, Michael (2010): Wandel von Arbeit - Wandel von Männlichkeiten. In: Österreichische Zeitschrift für Soziologie 35, 2, S. 89-103.

Lewis, Jane (2001): The decline of the male breadwinner model: implications for work and care. In: Social Politics, 8, 2, S. 152-169.

Lorber, Judith / Farrell, Susan A. (Hrsg.) (1991): The Social Construction of Gender. London: Sage.

Maihofer, Andrea (2002): Geschlecht und Sozialisation. Eine Problemskizze. In: Erwägen Wissen Ethik, 13 (1), S. 13-26.

Meuser, Michael (2006): Geschlecht und Männlichkeit. Soziologische Theorie und kulturelle Deutungsmuster. Wiesbaden: VS.

Meuser, Michael (2010): Geschlecht, Macht, Männlichkeit - Strukturwandel von Erwerbsarbeit und hegemoniale Männlichkeit. In: Erwägen - Wissen - Ethik 21, 3, S. 325-336.

McRobbie, Angela (2010): Top Girls. Feminismus und der Aufstieg des neoliberalen Geschlechterregimes. Wiesbaden: VS.

Michalitsch, Gabriele (2006): Die neoliberale Domestizierung des Subjekts. Von den Leidenschaften zum Kalkül. Frankfurt a.M.: Campus.

Michalitsch, Gabriele (2008): Selbstregulierte Subjekte. Privatisierung und Geschlechter-Regierung, in: Bidwell-Steiner, Marlen / Wagner, Ursula (Hrsg.): Freiheit und Geschlecht. Offene Beziehungen, prekäre Verhältnisse. Innsbruck: Studienverlag, S. 63-75.

Moser, Vera / Pinhard, Inga (Hrsg.) (2010): Care - Wer sorgt für wen? Jahrbuch Frauen- und Geschlechterforschung in der Erziehungswissenschaft, Bd. 6. Opladen: Barbara Budrich.

Moser, Vera / Rendtorff, Barbara (Hrsg.) (2012): Riskante Leben? Geschlechterordnungen in der reflexiven Moderne. Jahrbuch Frauen- und Geschlechterforschung in der Erziehungswissenschaft, Bd. 8. Opladen: Barbara Budrich. 
Nickel, Hildegard Maria (2012): Individualisierung und Subjektivierung aus der Geschlechterperspektive: Riskante Chancen. In: Moser, Vera / Rendtorff, Barbara (Hrsg.): Riskante Leben? A.a.O., S. 15-24.

Plößer, Melanie (2005): Dekonstruktion - Feminismus - Pädagogik. Vermittlungsansätze zwischen Theorie und Praxis. Königstein: Ulrike Helmer.

Pongratz, Hans J. / Voß, G. Günter (2003): Arbeitskraftunternehmer - Erwerbsorientierungen in entgrenzten Arbeitsformen, Berlin: Sigma.

Power, Nina (2011): Die eindimensionale Frau. Berlin: Merve.

Ptak, Ralf (2004): Vom Ordoliberalismus zur sozialen Marktwirtschaft. Stationen des Neoliberalismus in Deutschland. Opladen: Leske und Budrich.

Rendtorff, Barbara (2006): Erziehung und Geschlecht. Eine Einführung. Stuttgart: Kohlhammer.

Rothe, Daniela (2011): Lebenslanges Lernen als Programm: Eine diskursive Formation in der Erwachsenenbildung. Frankfurt a. M. / New York: Campus.

Sauer, Birgit (2001): Die Asche des Souveräns. Staat und Demokratie in der Geschlechterdebatte. Frankfurt a.M.: Campus.

Sauer, Dieter (2007): Vermarktlichung und Politik - Arbeitspolitik unter den Bedingungen Indirekter Steuerung. In: Peter, Gerd (Hrsg.): Grenzkonflikte der Arbeit Die Herausbildung einer neuen europäischen Arbeitspolitik. Hamburg: VSA, S. 202-217.

Scheu, Ursula (1977): Wir werden nicht als Mädchen geboren - wir werden dazu gemacht. Zur frühkindlichen Erziehung in unserer Gesellschaft. Frankfurt a. M.: Fischer.

Schmerl, Christiane (2006a): Einige Gedanken zur Sozialisation von Frauen. In: Schmerl, Christiane: Und sie bewegen sich doch ... Aus der Begegnung von Frauenbewegung und Wissenschaft. Tübingen: dgvt, S. 137-153.

Schmerl, Christiane (2006b): Männliche Reflexe, weibliche Reflexionen: Werbung mit Frauenbildern. In: Schmerl, Christiane: Und sie bewegen sich doch ... A.a.O., S. 289-305.

Scholz, Sylka (2004): Männlichkeit erzählen. Lebensgeschichtliche Identitätskonstrukte ostdeutscher Männer. Münster: Westfälisches Dampfboot.

Schröder, Gerhard / Blair, Tony (1999): Der Weg nach vorne für Europas Sozialdemokraten. Bonn / London.

Sennett, Richard (2000): Der flexible Mensch. Berlin: Siedler.

Tervooren, Anja (2006): Im Spielraum von Geschlecht und Begehren. Ethnographie der ausgehenden Kindheit. Weinheim: Juventa.

Thon, Christine (2008): Frauenbewegung im Wandel der Generationen. Eine Studie über Geschlechterkonstruktionen in biographischen Erzählungen. Bielefeld: transcript.

Thon, Christine (2012): Individualisierte Geschlechterordnungen? Feministische und hegemonieanalytische Kritik eines modernisierungstheoretischen Konzepts. In: Moser, Vera / Rendtorff, Barbara (Hrsg.): Riskante Leben? A.a.O., S. 27-43.

Thon, Christine (2015): ,Vereinbarkeit von Familie und Beruf“ - ein neoliberaler Diskurs? Familienpolitische und pädagogische Interventionen zur Herstellung von Subjekten der Vereinbarkeit. In: Walgenbach, Katharina / Stach, Anna (Hrsg): Geschlecht in gesellschaftlichen Transformationsprozessen. Opladen: Budrich, S. 131-143. 


\section{Bettina Dausien, Katharina Walgenbach}

Thürmer-Rohr, Christina (1983): Aus der Täuschung in die Ent-Täuschung. Zur Mittäterschaft von Frauen. In: Beiträge zur feministischen Theorie und Praxis, Heft 8/1983, S. 11-25.

Veith, Hermann (1996): Theorien der Sozialisation. Zur Rekonstruktion des modernen sozialisationstheoretischen Denkens. Frankfurt a. M. / New York: Campus.

Vereinigung der Bayrischen Wirtschaft (Hrsg.) (2007): Bildungsgerechtigkeit. Jahresgutachten 2007. Wiesbaden: VS.

Völker, Susanne (2009): ,Entsicherte Verhältnisse` - Impulse des Prekarisierungsdiskurses für eine geschlechtersoziologische Zeitdiagnose. In: Aulenbacher, Brigitte / Wetterer, Angelika (Hrsg.): Arbeit, Perspektiven und Diagnosen der Geschlechterforschung. Münster: Westfälisches Dampfboot, S. 268-286.

Walgenbach, Katharina (2014): Heterogenität - Intersektionalität - Diversity in der Erziehungswissenschaft. Opladen / Toronto: Babara Budrich / UTB.

Walgenbach, Katharina (2015): Geschlecht in gesellschaftlichen Transformationsprozessen. In: Walgenbach, Katharina / Stach, Anna (Hrsg): Geschlecht in gesellschaftlichen Transformationsprozessen. A.a.O., S. 21-51.

Walgenbach, Katharina / Stach, Anna (Hrsg.) (2015): Geschlecht in gesellschaftlichen Transformationsprozessen. Opladen: Barbara Budrich.

West, Candace / Zimmerman, Don H. (1987): Doing Gender. In: Gender and Society 1 (2), S. 125-151.

Wetterer, Angelika (2003): Rhetorische Modernisierung. Das Verschwinden der Ungleichheit aus den zeitgenössischen Differenzen. In: Knapp, Gudrun-Axeli (Hrsg.): Achsen der Differenz. Münster: Westfälisches Dampfboot, S. 286-319.

Wetterer, Angelika (2010): Konstruktion von Geschlecht: Reproduktionsweisen der Zweigeschlechtlichkeit. In: Becker, Ruth / Kortendiek, Beate (Hrsg.): Handbuch Frauen- und Geschlechterforschung (3. Aufl.). A.a.O., S. 127-136.

Young, Brigitte (1998): Genderregime und Staat in der globalen Netzwerkökonomie. In: Prokla 111, 28, 2, S. 175-199.

Zeitschrift für Soziologie der Erziehung und Sozialisation (ZSE) (2002): SelbstSozialisation in der Diskussion. Schwerpunkt, Heft 2/2002. 